\title{
CP Violation and Neutrino Masses and Mixings from Quark Mass Hierarchies
}

\author{
Wilfried Buchmüller \\ Deutsches Elektronen-Synchrotron DESY, Hamburg, Germany \\ Laura Covi \\ Deutsches Elektronen-Synchrotron DESY, Hamburg, Germany \\ David Emmanuel-Costa \\ CFTP, Departamento de Fisica, Istituto Superior Tecnico, Lisbon, Portugal
}

\author{
Sören Wiesenfeldt \\ Department of Physics, University of Illinois at Urbana-Champaign, USA
}

\begin{abstract}
We study the connection between quark and lepton mass matrices in a supersymmetric SO(10) GUT model in six dimensions, compactified on an orbifold. The physical quarks and leptons are mixtures of brane and bulk states. This leads to a characteristic pattern of mass matrices and high-energy CP violating phases. The hierarchy of up and down quark masses determines the CKM matrix and most charged lepton and neutrino masses and mixings. The small hierarchy of neutrino masses is a consequence of the mismatch of the up and down quark mass hierarchies. The effective CP violating phases in the quark sector, neutrino oscillations and leptogenesis are unrelated. In the neutrino sector we can accomodate naturally $\sin \theta_{23} \sim 1, \sin \theta_{13} \lesssim 0.1$ and $m_{1} \lesssim m_{2} \sim \sqrt{\Delta m_{\text {sol }}^{2}}<m_{3} \sim \sqrt{\Delta m_{\text {atm }}^{2}}$
\end{abstract}

Keywords: CP violation, Field Theories in Higher Dimensions, GUT, Neutrino Physics. 


\section{Contents}

1. Introduction 1

2. $\mathrm{SO}(10)$ Unification in six dimensions 2

3. CP violation in the quark sector 8

14. CP violation in the leptonic sector 11

4.1 Seesaw Mechanism and Effective Mass Matrix

4.2 Neutrinoless Double Beta Decay $(0 \nu \beta \beta)$

4.3 CP Violation in Neutrino Oscillations

4.4 Leptogenesis 18

5. Conclusions 20

A. Mass matrices 22

A.1 Down Quarks and Charged Leptons 23

A.2 Neutrinos 26

B. CP Violation and Weak Basis Invariants 35

\section{Introduction}

Grand unified theories (GUTs) appear to be the most promising framework [1,2] to address the still challenging question of quark and lepton masses and mixings. During the past years new results from neutrino physics have shed new light on this problem, and the large differences between the mass hierarchies and mixing angles of quarks, charged leptons and neutrinos impose strong constraints on unified extensions of the Standard Model (SM) [3,4]. Massive neutrinos are most easily incorporated in theories with right-handed neutrinos, which leads to $\mathrm{SO}(10)$ as preferred GUT gauge group $[5,6]$.

Higher-dimensional theories offer new possibilities to describe gauge symmetry breaking, the notorious doublet-triplet splitting and also fermion masses. A simple and elegant scheme is provided by orbifold compactifications which have recently been considered for GUT models in five and six dimensions [7-12]. In this paper we analyse in detail the connection between quark and lepton mass matrices in the 
six-dimensional (6D) GUT model suggested in [13], for which also proton decay [14], supersymmetry breaking [15] and gauge coupling unification [16] have been studied. An alternative $\mathrm{SO}(10)$ model in five and six dimensions has previously been studied in [17]. For a recent discussion of CP violation in a 5D orbifold GUT model, see [18].

An important ingredient of orbifold GUTs is the presence of split bulk multiplets whose mixings with complete GUT multiplets, localised at the fixed points, can significantly modify ordinary GUT mass relations. This extends the known mechanism of mixing with vectorlike multiplets [19-21]. Such models have a large mixing of left-handed leptons and right-handed down quarks, while small mixings of the lefthanded down quarks. In this way large mixings in the leptonic charged current are naturally reconciled with small CKM mixings in the quark current.

Our model of quark and lepton masses and mixings relates different orders of magnitude whereas factors $\mathcal{O}(1)$ remain undetermined. Hence, we can only discuss qualitative features of quark and lepton mass matrices. Recently, orbifold compactifications of the heterotic string have been constructed which can account for the standard model in four dimensions and which have a six-dimensional GUT structure as intermediate step very similar to familiar orbifold GUT models [22-24]. In such models the currently unknown $\mathcal{O}(1)$ factors are in principle calculable, which would then allow for quantitative predictions.

The goal of the present paper is twofold: As a typical example, we first study the model [13] in more detail and explicitly compute the mass eigenstates, masses and mixing angles. Second, we investigate the question of $\mathrm{CP}$ violation, both in the quark and lepton sector and possible connections between the two. In previous studies, CP violation has mostly been neglected assuming that, barring fortunate cancellations, the phases and mixings are practically independent. Nevertheless this question and the flavour structure are strongly interconnected, and we will see that a specific pattern of mass matrices can give a distinct signature also in the $\mathrm{CP}$ violation invariants.

This paper is organised as follows: In Section 2 we describe the 6D orbifold GUT model and the diagonalisation of the mass matrices defining the low energy SM fermions. In Section 3 we discuss the CP violation in the quark sector, whereas Section 14 is devoted to the $\mathrm{CP}$ violation in the leptonic sector. Conclusions are given in Section 0 . Two appendices provide details to the computation of the mass eigenstates and $\mathrm{CP}$ violation in extensions of the SM.

\section{2. $\mathrm{SO}(10)$ Unification in six dimensions}

We study an $\mathrm{SO}(10)$ GUT model in $6 \mathrm{D}$ with $N=1$ supersymmetry compactified on the orbifold $\mathbb{T}^{2} /\left(\mathbb{Z}_{2}^{\mathrm{I}} \times \mathbb{Z}_{2}^{\mathrm{PS}} \times \mathbb{Z}_{2}^{\mathrm{GG}}\right)[11,12]$. The theory has four fixed points, $O_{\mathrm{I}}$, $O_{\mathrm{PS}}, O_{\mathrm{GG}}$ and $O_{\mathrm{fl}}$, located at the four corners of a 'pillow' corresponding to the two compact dimensions (cf. Fig. 目). The extended supersymmetry is broken at all fixed 


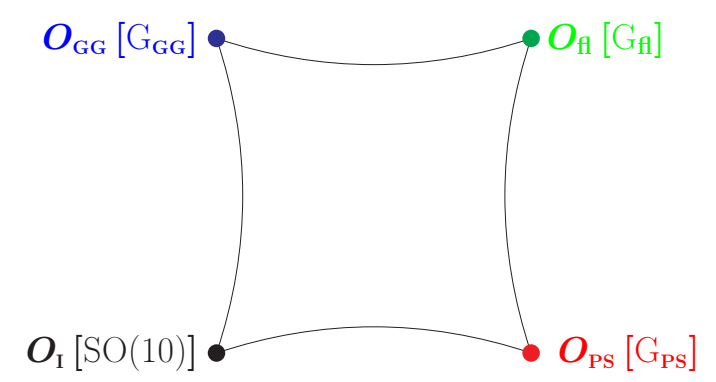

Figure 1: The three $\mathrm{SO}(10)$ subgroups at the corresponding fixed points (branes) of the orbifold $\mathbb{T}^{2} /\left(\mathbb{Z}_{2}^{I} \times \mathbb{Z}_{2}^{\mathrm{PS}} \times \mathbb{Z}_{2}^{\mathrm{GG}}\right)$.

points; in addition, the gauge group $\mathrm{SO}(10)$ is broken to its three subgroups $\mathrm{G}_{\mathrm{PS}}=$ $\mathrm{SU}(4) \times \mathrm{SU}(2) \times \mathrm{SU}(2) ; \mathrm{G}_{\mathrm{GG}}=\mathrm{SU}(5) \times \mathrm{U}(1)_{X}$; and flipped $\mathrm{SU}(5), \mathrm{G}_{\mathrm{fl}}=\mathrm{SU}(5)^{\prime} \times$ $\mathrm{U}(1)^{\prime}$, at $O_{\mathrm{PS}}, O_{\mathrm{GG}}$ and $O_{\mathrm{fl}}$, respectively. The intersection of all these GUT groups yields the standard model group with an additional $\mathrm{U}(1)$ factor, $\mathrm{G}_{\mathrm{SM}^{\prime}}=\mathrm{SU}(3) \times$ $\mathrm{SU}(2) \times \mathrm{U}(1)_{Y} \times \mathrm{U}(1)_{Y^{\prime}}$, as unbroken gauge symmetry below the compactification scale.

The field content of the theory is strongly constrained by imposing the cancellation of irreducible bulk and brane anomalies [25]. The model proposed in Ref. [13] contains three spinors $\psi_{i}(16), i=1 \ldots 3$, as brane fields as well as six vectorial fields $H_{j}(10), j=1 \ldots 6$, and two pairs of spinors, $\Phi(16)+\Phi^{c}(\overline{16})$ and $\phi(16)+\phi^{c}(\overline{16})$ as bulk hypermultiplets.

The massless zero modes $N(\Phi)$ and $N^{c}\left(\Phi^{c}\right)$ acquire vacuum expectation values (vevs), $v_{N}=\langle N\rangle=\left\langle N^{c}\right\rangle$, breaking $B-L$ and thus $\mathrm{G}_{\mathrm{SM}^{\prime}}$ to $\mathrm{G}_{\mathrm{SM}}$. The breaking scale is close to the compactification scale so that $v_{N}^{2} / M_{*} \sim 10^{14} \mathrm{GeV}$, where $M_{*}$ is the cutoff of the $6 \mathrm{D}$ theory. At the weak scale, the doublets $H_{d}\left(H_{1}\right)$ and $H_{u}\left(H_{2}\right)$ acquire vevs, $v_{1}=\left\langle H_{d}\right\rangle$ and $v_{2}=\left\langle H_{u}\right\rangle$, breaking the electroweak symmetry.

The three sequential 16-plets are located on the three branes where $\mathrm{SO}(10)$ is broken to its three GUT subgroups; in particular, we place $\psi_{1}$ at $O_{\mathrm{GG}}, \psi_{2}$ at $O_{\mathrm{f}}$ and $\psi_{3}$ at $O_{\mathrm{PS}}$. The parities of $H_{5}, H_{6}, \phi$, and $\phi^{c}$ are chosen such that their zero modes,

$$
L(\phi)=\left(\begin{array}{c}
\nu_{4} \\
e_{4}
\end{array}\right), \quad L^{c}\left(\phi^{c}\right)=\left(\begin{array}{c}
\nu_{4}^{c} \\
e_{4}^{c}
\end{array}\right), \quad d_{4}^{c}\left(H_{5}\right), \quad d_{4}\left(H_{6}\right)
$$

have the quantum numbers of a lepton doublet and antidoublet as well as anti-down and down-quark singlets, respectively. Both $L(\phi)$ and $L^{c}\left(\phi^{c}\right)$ are $\mathrm{SU}(2)_{L}$ doublets. Together these zero modes act as a fourth vectorial generation of down quarks and leptons.

The three 'families' $\psi_{i}$ are separated by distances large compared to the cutoff scale $M_{*}$. Hence, they can only have diagonal Yukawa couplings with the bulk Higgs fields; direct mixings are exponentially suppressed. The brane fields, however, can mix with the bulk zero modes for which we expect no suppression. These mixings 
take place only among left-handed leptons and right-handed down quarks, leading to a characteristic pattern of mass matrices [13,14].

The mass terms assume the characteristic form,

$$
W=u_{i} m_{i}^{u} u_{i}^{c}+d_{\alpha} m_{\alpha \beta}^{d} d_{\beta}^{c}+e_{\alpha}^{c} m_{\alpha \beta}^{e} e_{\beta}+\nu_{\alpha}^{c} m_{\alpha \beta}^{D} \nu_{\beta}+\frac{1}{2} \nu_{i}^{c} m_{i}^{N} \nu_{i}^{c},
$$

where latin indices only span 1,2,3, while greak indices include the forth generation states. The up quark and Majorana neutrino mass matrices, $m^{u}$ and $m^{N}$, are diagonal $3 \times 3$ matrices,

$$
m^{u}=\left(\begin{array}{ccc}
h_{11}^{u} v_{2} & 0 & 0 \\
0 & h_{22}^{u} v_{2} & 0 \\
0 & 0 & h_{33}^{u} v_{2}
\end{array}\right), \quad m^{N}=\left(\begin{array}{ccc}
h_{11}^{N} \frac{v_{N}^{2}}{M_{*}} & 0 & 0 \\
0 & h_{22}^{N} \frac{v_{N}^{2}}{M_{*}} & 0 \\
0 & 0 & h_{33}^{N} \frac{v_{N}^{2}}{M_{*}}
\end{array}\right)
$$

Since $\nu_{4}^{c}$ is part of an $\mathrm{SU}(2)_{L}$ doublet, it cannot couple to the other SM singlets in $\psi_{i}$ via the $B-L$ breaking field. Furthermore, there is no other coupling giving it a direct Majorana mass.

The Dirac mass matrices of down quarks, charged leptons and neutrinos, $m^{d}, m^{e}$ and $m^{D}$, respectively, are $4 \times 4$ matrices instead, due to the mixing with the bulk field zero modes,

$$
\begin{aligned}
m^{d} & =\left(\begin{array}{cccc}
h_{11}^{d} v_{1} & 0 & 0 & g_{1}^{d} \frac{v_{N}}{M_{*}} v_{1} \\
0 & h_{22}^{d} v_{1} & 0 & g_{2}^{d} \frac{v_{N}}{M_{*}} v_{1} \\
0 & 0 & h_{33}^{d} v_{1} & g_{3}^{d} \frac{v_{N}}{M_{*}} v_{1} \\
f_{1} v_{N} & f_{2} v_{N} & f_{3} v_{N} & M^{d}
\end{array}\right), \\
m^{e} & =\left(\begin{array}{cccc}
h_{11}^{d} v_{1} & 0 & 0 & h_{14}^{e} v_{1} \\
0 & h_{22}^{e} v_{1} & 0 & h_{24}^{e} v_{1} \\
0 & 0 & h_{33}^{d} v_{1} & h_{34}^{e} v_{1} \\
M_{1}^{l} & M_{2}^{l} & M_{3}^{l} & M_{4}^{l}
\end{array}\right), \quad m^{D}=\left(\begin{array}{cccc}
h_{11}^{D} v_{2} & 0 & 0 & h_{14}^{D} v_{2} \\
0 & h_{22}^{u} v_{2} & 0 & h_{24}^{D} v_{2} \\
0 & 0 & h_{33}^{u} v_{2} & h_{34}^{D} v_{2} \\
M_{1}^{l} & M_{2}^{l} & M_{3}^{l} & M_{4}^{l}
\end{array}\right),
\end{aligned}
$$

up to corrections $\mathcal{O}\left(v_{N}^{2} / M_{*}^{2}\right)$. The diagonal elements satisfy four GUT relations which correspond only to the local unbroken groups, i.e., SU(5), flipped SU(5) and Pati-Salam subgroups of $\mathrm{SO}(10)$. The hypothesis of a universal strength of Yukawa couplings at each fixpoint leads to the identification of the diagonal and off-diagonal elements of $m^{u} / \tan \beta, m^{d}, m^{e}$, and $m^{D} / \tan \beta$, where $\tan \beta=v_{2} / v_{1}$, up to coefficients of order one. This implies an approximate top-bottom unification with large $\tan \beta$ and a parametrisation of quark and lepton mass hierarchies in terms of the six parameters $\mu_{i}$ and $\widetilde{\mu}_{i}$.

The crucial feature of the matrices $m^{d}, m^{e}$ and $m^{D}$ are the mixings between the six brane states and the two bulk states. The first three rows of the matrices are proportional to the electroweak scale. The corresponding Yukawa couplings have to be hierarchical in order to obtain a realistic spectrum of quark and lepton masses. 
This corresponds to different strengths of the Yukawa couplings at the different fixed points of the orbifold. The fourth row, proportional to $M^{d}, M^{l}$ and $v_{N}$, is of order the unification scale and, we assume, non-hierarchical.

The mass matrices $m^{d}, m^{e}$ and $m^{D}$ are of the common form

$$
m=\left(\begin{array}{cccc}
\mu_{1} & 0 & 0 & \widetilde{\mu}_{1} \\
0 & \mu_{2} & 0 & \widetilde{\mu}_{2} \\
0 & 0 & \mu_{3} & \widetilde{\mu}_{3} \\
\widetilde{M_{1}} & \widetilde{M}_{2} & \widetilde{M}_{3} & \widetilde{M}_{4}
\end{array}\right),
$$

where $\mu_{i}, \widetilde{\mu}_{i}=\mathcal{O}\left(v_{1,2}\right)$ and $\widetilde{M}_{i}=\mathcal{O}\left(M_{\mathrm{GUT}}\right)$. This matrix can be diagonalised using the unitary matrices

$$
m=U_{4} U_{3} D V_{3}^{\dagger} V_{4}^{\dagger}
$$

where the matrices $U_{4}$ and $V_{4}$ single out the heavy mass eigenstate, that can then be integrated away, while $U_{3}$ and $V_{3}$ act only on the SM flavour indices and perform the final diagonalisation also in the $3 \times 3$ subspace. The explicit expressions for the mixing matrices and the mass eigenstates are given in Appendix A.

The parameters in the matrix Eq. (2.4) are generally complex; however, we can absorb seven phases with appropriate field redefinitions and choose the remaining three physical phases to be contained into the diagonal parameters $\mu_{i}$,

$$
m=\left(\begin{array}{cccc}
\left|\mu_{1}\right| e^{i \theta_{1}} & 0 & 0 & \widetilde{\mu}_{1} \\
0 & \left|\mu_{2}\right| e^{i \theta_{2}} & 0 & \widetilde{\mu}_{2} \\
0 & 0 & \left|\mu_{3}\right| e^{i \theta_{3}} & \widetilde{\mu}_{3} \\
\widetilde{M}_{1} & \widetilde{M}_{2} & \widetilde{M}_{3} & \widetilde{M}_{4}
\end{array}\right) .
$$

This is the maximal number of physical phases for four generations of Dirac fermions, given as usual by $(n-1)(n-2) / 2$ for $n$ generations, so our texture above does not reduce the $\mathrm{CP}$ violation from the typical $n=4$ case. We will see that the phases survive in the low energy parameters, but that only one combination defines the single phase characteristic of three generations.

With this choice, the matrix $V_{4}$ is real, while $U_{4}$ contains complex parameters; however, the imaginary part is suppressed by $\left|\mu_{i}\right| / \widetilde{M}$ so that their effect on the low energy $\mathrm{CP}$ violation is negligible as long as the mass of the heavy eigenstate is large compared to the electroweak scale. From the unification of the gauge couplings, we expect indeed $\widetilde{M}$ to be of the order of the GUT scale [16]. Then the discussion of the low energy $\mathrm{CP}$ violation, which would in general be characterised by many $\mathrm{CP}$ invariants [26, 27], reduces to the case of three light generations (see Appendix B).

The effective mass matrix is given by $\widehat{m}$, the $3 \times 3$ part of

$$
m^{\prime}=U_{4}^{\dagger} m V_{4}=\left(\begin{array}{cc}
\widehat{m} & 0 \\
0 & \widetilde{M}
\end{array}\right)+\mathcal{O}\left(\frac{v^{2}}{\widetilde{M}^{2}}\right), \quad \widehat{m}=\left(\begin{array}{c}
\mu_{1}\left(V_{4}\right)_{1 j}+\widetilde{\mu}_{1}\left(V_{4}\right)_{4 j} \\
\mu_{2}\left(V_{4}\right)_{2 j}+\widetilde{\mu}_{2}\left(V_{4}\right)_{4 j} \\
\mu_{3}\left(V_{4}\right)_{3 j}+\widetilde{\mu}_{3}\left(V_{4}\right)_{4 j}
\end{array}\right) ;
$$


in terms of the parameters in Eq. (2.4), it reads

$$
\widehat{m}=\left(\begin{array}{cccc}
\mu_{1} \frac{\widetilde{M}_{4}}{\sqrt{\widetilde{M}_{1}^{2}+\widetilde{M}_{4}^{2}}}-\widetilde{\mu}_{1} \frac{\widetilde{M}_{1}}{\sqrt{\widetilde{M}_{1}^{2}+\widetilde{M}_{4}^{2}}} & 0 & -\mu_{1} \frac{\widetilde{M}_{1} \sqrt{\widetilde{M}_{2}^{2}+\widetilde{M}_{3}^{2}}}{\sqrt{\widetilde{M}_{1}^{2}+\widetilde{M}_{4}^{2}}}-\widetilde{\mu}_{1} \frac{\widetilde{M}_{4} \sqrt{\widetilde{M}_{2}^{2}+\widetilde{M}_{3}^{2}}}{\sqrt{\widetilde{M}_{1}^{2}+\widetilde{M}_{4}^{2}}} \\
-\widetilde{\mu}_{2} \frac{\widetilde{M}_{1}}{\sqrt{\widetilde{M}_{1}^{2}+\widetilde{M}_{4}^{2}}} & \mu_{2} \frac{\widetilde{M}_{3}}{\sqrt{\widetilde{M}_{2}^{2}+\widetilde{M}_{3}^{2}}} & \mu_{2} \frac{\widetilde{M_{2}} \sqrt{\widetilde{M}_{1}^{2}+\widetilde{M}_{4}^{2}}}{\widetilde{M} \sqrt{\widetilde{M}_{2}^{2}+\widetilde{M}_{3}^{2}}}-\widetilde{\mu}_{2} \frac{\widetilde{M}_{4} \sqrt{\widetilde{M}_{2}^{2}+\widetilde{M}_{3}^{2}}}{\widetilde{\widetilde{M}_{1}^{2}+\widetilde{M}_{4}^{2}}} \\
-\widetilde{\mu}_{3} \frac{\widetilde{M}_{1}}{\sqrt{\widetilde{M}_{1}^{2}+\widetilde{M}_{4}^{2}}} & -\mu_{3} \frac{\widetilde{M}_{2}}{\sqrt{\widetilde{M}_{2}^{2}+\widetilde{M}_{3}^{2}}} & \mu_{3} \frac{\widetilde{M} \sqrt{\widetilde{M}_{1}^{2}+\widetilde{M}_{4}^{2}}}{\widetilde{M} \sqrt{\widetilde{M}_{2}^{2}+\widetilde{M}_{3}^{2}}}-\widetilde{\mu}_{3} \frac{\widetilde{M} \sqrt{\widetilde{M}_{2}^{2}+\widetilde{M}_{3}^{2}}}{\widetilde{M} \sqrt{\widetilde{M}_{1}^{2}+\widetilde{M}_{4}^{2}}}
\end{array}\right) .
$$

As any matrix, $\widehat{m}$ can be transformed into upper triangular form just by basis redefinition on the right,

$$
\bar{m}=\widehat{m} \widehat{V}_{3}=\left(\begin{array}{ccc}
\gamma \bar{\mu}_{1} & \bar{\mu}_{1} & \beta \bar{\mu}_{1} \\
0 & \bar{\mu}_{2} & \alpha \bar{\mu}_{2} \\
0 & 0 & \bar{\mu}_{3}
\end{array}\right)
$$

This form is particularly suitable in the case of the down quarks, where $\widehat{V}_{3}$ acts on the right-handed quarks and disappears from the low energy Lagrangian due to the absence of right-handed current interactions. Note that we can reshuffle the phases, reabsorbing three of them into the unitary transformation $\widehat{V}_{3}$, but we are still left with three complex parameters. We can exploit this freedom to obtain real diagonal elements $\bar{\mu}_{2}, \bar{\mu}_{3}$ and $\gamma \bar{\mu}_{1}$, while $\alpha, \beta$, and $\bar{\mu}_{1}$ remain complex.

On the other hand, we can still redefine two phases on the left-hand side, keeping an overall phase free, with a diagonal matrix

$$
P_{L 3}=\operatorname{diag}\left(e^{-i \zeta_{1}}, e^{-i \zeta_{2}}, 1\right)
$$

This transformation allows us to shift the phase of $\bar{\mu}_{1}$ into $\gamma$, which will be convenient later in the limit where $\gamma$ vanishes. Again, such a phase shift does not reduce the number of complex parameters in the down quark matrix, which remains three. Moreover, this reparametrisation does not change the CKM matrix, since the up quark mass matrix is diagonal and so such phase transformation can be compensated by an identical one for both $u_{i}$ and $u_{i}^{c}$.

The matrix $\widehat{V}_{3}$ differs from the upper $3 \times 3$ part of the diagonalising matrix $V_{3}=\widehat{V}_{3} V_{3}^{\prime}$; however, they are very similar in the hierarchical case. The relation between these two can be found in Appendix A, together with the general expression for $U_{3}$, the $3 \times 3$ part of which is the CKM matrix.

For the leptons, it is the matrix $V_{4} V_{3}$ that acts on the left-handed states, so the mismatch between the charged leptons and neutrinos (see Eq. (2.30)) basis appears in the charged current interaction and the definition of the flavour neutrino eigenstates. However, the matrix $V_{4}$ which contains large mixing angles and rotates away the heavy eigenstate is the same for charged leptons and neutrinos since the heavy state is an $\mathrm{SU}(2)_{L}$ doublet. Therefore the PMNS matrix will be given only by the mismatch between the $\widehat{V}_{3} \simeq V_{3}$ matrices for charged leptons and neutrinos. 
The complete expressions for the parameters in $\bar{m}$ are given in Appendix $\mathrm{A}$; in this section, we will only consider the limit of small $\mu_{1}$ as well as small $\widetilde{\mu}_{1}$ and/or $\mu_{2}$. For $\mu_{1}=\widetilde{\mu}_{1}=0$, the first row simply vanishes, whereas for $\mu_{1}=\mu_{2}=0$, the two first rows of the mass matrix are aligned (see Eq. (2.6)). Therefore both cases correspond to vanishing down-quark and electron mass.

Since $\widetilde{\mu}_{1} / \widetilde{\mu}_{2}$ gives $V_{u s}$, we focus on the case $\mu_{1}=\mu_{2}=0$, where $^{1}$

$$
\begin{aligned}
& \alpha=\beta=\frac{\widetilde{\mu}_{2}}{\bar{\mu}_{2}}\left(\frac{\widetilde{\mu}_{3}}{\bar{\mu}_{3}}-\frac{\widetilde{M}_{4}}{\widetilde{M}} \frac{\mu_{3}^{*} \widetilde{M}_{3}+\widetilde{\mu}_{3} \widetilde{M}_{4}}{\bar{\mu}_{3} \widetilde{M}}\right), \\
& \frac{\bar{\mu}_{1}}{\widetilde{\mu}_{1}}=\frac{\bar{\mu}_{2}}{\widetilde{\mu}_{2}}=\frac{\left|\mu_{3}\right|}{\bar{\mu}_{3}} \frac{\sqrt{\widetilde{M}_{1}^{2}+\widetilde{M}_{2}^{2}}}{\widetilde{M}} .
\end{aligned}
$$

The eigenvalues of the heavier states are given by

$$
\begin{aligned}
& m_{b}^{2}=\bar{\mu}_{3}^{2}, \\
& m_{s}^{2}=\bar{\mu}_{2}^{2}+\left|\bar{\mu}_{1}\right|^{2}=\bar{\mu}_{2}^{2}\left(1+\frac{\widetilde{\mu}_{1}^{2}}{\widetilde{\mu}_{2}^{2}}\right) \sim \bar{\mu}_{2}^{2}, \quad \text { where } \frac{\widetilde{\mu}_{1}}{\widetilde{\mu}_{2}} \sim V_{u s} .
\end{aligned}
$$

In this limit, only one single physical CP violating phase survives, even in the $4 \times 4$ picture; it is contained in $\mu_{3}$ and so in $\alpha$ and $\beta$ (see Eq. (2.10)). We will see, however, that this single phase is not sufficient to have low-energy $\mathrm{CP}$ violation.

The down-quark mass is indeed very small, so we will use these expressions as the order zero approximation, together with the corrections proportional to $\left|\mu_{2}\right| / \bar{\mu}_{2}$, which determine the masses of the down-quark and the electron. Our expansion parameter will therefore be of the order of the mass ratio of the down and strangequark, $m_{d} / m_{s}$. In fact, for $\left|\mu_{1}\right| \ll\left|\mu_{2}\right|$ we have at leading order

$$
m_{d}=\gamma \bar{\mu}_{1} \simeq \widetilde{\mu}_{1} \frac{\left|\mu_{2}\right|}{\bar{\mu}_{2}} \frac{\left|\mu_{3}\right|}{\bar{\mu}_{3}} \simeq\left|\mu_{2}\right| \frac{\left|\bar{\mu}_{1}\right|}{\bar{\mu}_{2}} \simeq V_{u s}\left|\mu_{2}\right|,
$$

so our expansion parameter is

$$
\frac{\left|\mu_{2}\right|}{\bar{\mu}_{2}} \simeq \frac{m_{d}}{m_{s} V_{u s}} \sim 0.23 .
$$

The mass ratio of electron and muon is much smaller than the ratio of down and strange quark. This implies $\left(\mu_{2} \widetilde{\mu}_{1} / \widetilde{\mu}_{2}^{2}\right)_{e} \ll\left(\mu_{2} \widetilde{\mu}_{1} / \widetilde{\mu}_{2}^{2}\right)_{d}$. Assuming that the difference is due to the smallest matrix elements, this indicates $\left(\mu_{2}\right)_{e} /\left(\mu_{2}\right)_{d} \ll 1$ and/or $\left(\widetilde{\mu}_{1}\right)_{e} /\left(\widetilde{\mu}_{1}\right)_{d} \ll 1$ for $\left(\widetilde{\mu}_{2}\right)_{e} \simeq\left(\widetilde{\mu}_{2}\right)_{d}$. This fact can easily be accommodated, as we see in Eqs. (2.3): the presence of the second generation on the flipped $\mathrm{SU}(5)$

\footnotetext{
${ }^{1}$ As mentioned above, it is instructive to choose the basis in which $\bar{\mu}_{1}$ is real and the vanishing parameter $\gamma \bar{\mu}_{1}$ complex. Then it is obvious that we are left with only two complex parameters in $\bar{m}$, namely $\alpha$ and $\beta$, containing the same phase.
} 
brane leads to different values of $\mu_{2}$ for the down quarks and charged leptons and the parameter $\widetilde{\mu}_{1}$ stems from different couplings in the superpotential.

While we derived the fermion mass matrices (2.3) within a specific model, they can also arise in other models, where additional matter is present at the GUT (or compactification) scale. Thus we could take these matrices as a starting point for the following discussion, leaving open the question of their origin.

\section{3. $\mathrm{CP}$ violation in the quark sector}

We will first consider the $\mathrm{CP}$ violation in the quark sector. As we have seen in the previous section, our effective $3 \times 3$ down quark mass matrix contains three phases as a remnant of the original $4 \times 4$ matrix, with the dominant complex element being $\alpha \bar{\mu}_{2}$. We will now derive the combination of the three phases, which plays the role of the CKM phase.

To describe $\mathrm{CP}$ violation for three generations, as is the case in the SM, it is convenient to use the Jarlskog invariant [28], $J_{q}$, which is given by

$$
6 i \Delta \mathscr{M}_{u}^{2} \Delta \mathscr{M}_{d}^{2} J_{q}=\operatorname{tr}\left[H_{u}, H_{d}\right]^{3}=6 \operatorname{Im} \operatorname{tr}\left(H_{u}^{2} H_{d}^{2} H_{u} H_{d}\right) \text {, }
$$

where $H=m m^{\dagger}$ and

$$
\Delta \mathscr{M}^{2}=\left(m_{3}^{2}-m_{2}^{2}\right)\left(m_{3}^{2}-m_{1}^{2}\right)\left(m_{2}^{2}-m_{1}^{2}\right)
$$

note that $\Delta \mathscr{M}^{2}$ has mass-dimension six. In our model, the up quark mass matrix is diagonal, as is $H_{u}$. Then the invariant strongly simplifies and reads

$$
J_{q}=\frac{\operatorname{Im}\left(H_{d}^{12} H_{d}^{23} H_{d}^{31}\right)}{\Delta \mathscr{M}_{d}^{2}} .
$$

It is clear from this expression, that any diagonal phase transformation of $m$ on the left does not have any effect on the Jarlskog invariant.

As discussed in Appendix $B$, we can use the effective $3 \times 3$ mass matrix $H_{d}^{\text {eff }}=\widehat{m} \widehat{m}^{\dagger}=\bar{m} \bar{m}^{\dagger}$. By means of Eq. (2.8), we obtain

$$
H_{d}^{\mathrm{eff}}=\left(\begin{array}{ccc}
\left|\bar{\mu}_{1}\right|^{2}\left(1+|\beta|^{2}+|\gamma|^{2}\right) & \bar{\mu}_{1} \bar{\mu}_{2}\left(1+\alpha^{*} \beta\right) & \bar{\mu}_{1} \bar{\mu}_{3} \beta \\
\bar{\mu}_{1}^{*} \bar{\mu}_{2}\left(1+\alpha \beta^{*}\right) & \bar{\mu}_{2}^{2}\left(1+|\alpha|^{2}\right) & \bar{\mu}_{2} \bar{\mu}_{3} \alpha \\
\bar{\mu}_{1}^{*} \bar{\mu}_{3} \beta^{*} & \bar{\mu}_{2} \bar{\mu}_{3} \alpha^{*} & \bar{\mu}_{3}^{2}
\end{array}\right),
$$

where $\bar{\mu}_{2}$ and $\bar{\mu}_{3}$ are real parameters, as displayed in Eqs. (A.4). Then we have

$$
\begin{aligned}
\operatorname{Im}\left[\left(H_{d}^{\text {eff }}\right)^{12}\left(H_{d}^{\text {eff }}\right)^{23}\left(H_{d}^{\text {eff }}\right)^{31}\right] & =\left|\bar{\mu}_{1}\right|^{2} \bar{\mu}_{2}^{2} \bar{\mu}_{3}^{2} \operatorname{Im} \alpha \beta^{*}\left(1+\alpha^{*} \beta\right) \\
& =\left|\bar{\mu}_{1}\right|^{2} \bar{\mu}_{2}^{2} \bar{\mu}_{3}^{2} \operatorname{Im} \alpha \beta^{*} \\
& =\bar{\mu}_{2} \bar{\mu}_{3}^{2} \operatorname{Im}\left[\left(\alpha \bar{\mu}_{2}\right)\left(\beta \bar{\mu}_{1}\right)^{*} \bar{\mu}_{1}\right] .
\end{aligned}
$$


We see that the Jarlskog invariant is always independent of the argument of $\gamma$ and it vanishes in the limit $\mu_{1}, \widetilde{\mu}_{1} \rightarrow 0$ such that $\bar{\mu}_{1}=0$. As we might expect, $J_{q}$ vanishes for $\alpha=\beta$ as well, i.e., in the limit $\mu_{1}, \mu_{2} \rightarrow 0$.

So the presence of a single phase in $\alpha$ is not sufficient to give $\mathrm{CP}$ violation in the low energy: this phase cancels out in the Jarlskog invariant. This effect stems from the alignment of the vectors in flavour space; however, even in the case of vanishing first generation mass, the corresponding eigenvector does not decouple from the other two and the mixing matrix does not reduce to the two-generational case. In fact, the CKM matrix is given by (see Appendix A $)^{2}$

$$
V_{\mathrm{CKM}}\left(m_{d}=0\right) \simeq\left(\begin{array}{ccc}
1 & \frac{\bar{\mu}_{1}}{\bar{\mu}_{2}} & \frac{\bar{\mu}_{1} \alpha}{\bar{\mu}_{3}} \\
-\frac{\bar{\mu}_{1}^{*}}{\bar{\mu}_{2}} & 1 & \frac{\bar{\mu}_{2 \alpha}}{\bar{\mu}_{3}} \\
0 & -\frac{\bar{\mu}_{2} \alpha^{*}}{\bar{\mu}_{3}} & 1
\end{array}\right), \quad U_{3}=\left(\begin{array}{cc}
V_{\mathrm{CKM}}^{\dagger} & 0 \\
0 & 1
\end{array}\right)
$$

Hence, we cannot conclude that the CP effects disappear due to the reduction of the system to two generations, nor to the mass degeneracy between quarks. Instead the absence of low energy CP violation is caused by the particular texture of $m$ in exactly the same basis for the left-handed quark doublet, where the up quark matrix is diagonal. This feature is similar to the absence of CP violation in 4D $\mathrm{SO}(10)$ constructions, where a single ten-dimensional Higgs field generates fermionic masses, yielding a trivial CKM matrix. Note that there is still some CP violation effect arising from the dominant phase $\theta_{3}$ in $\mu_{3}$, but it is only apparent in the mixings involving the fourth heavy state.

Now, the down quark is not massless and the real physical case corresponds to non-zero $\mu_{1}, \mu_{2}$ and $\widetilde{\mu}_{1}$. From the up quark phenomenology, we know that $\mu_{1}: \mu_{2}$ is similar to the mass ratio of up and charm-quark [13]; in addition, $\widetilde{\mu}_{1}: \widetilde{\mu}_{2}$ is fixed by the Cabibbo angle. We will therefore focus on the linear terms in $\mu_{2}$ and keep $\mu_{1} \simeq 0$.

As is apparent in Eq. (3.5), contributions to $J_{q}$ come from the complex quantities $\alpha \bar{\mu}_{2}, \beta \bar{\mu}_{1}$, and $\bar{\mu}_{1}$; however, $\beta \bar{\mu}_{1}$ is independent of $\mu_{2}$ (see Eq. (A.4)),

$$
\beta \bar{\mu}_{1}=\widetilde{\mu}_{1}\left[\frac{\widetilde{\mu}_{3}}{\bar{\mu}_{3}}-\frac{\widetilde{M}_{4}}{\widetilde{M}} \frac{\widetilde{\mu}_{3} \widetilde{M}_{4}+\mu_{3}^{*} \widetilde{M}_{3}}{\bar{\mu}_{3} \widetilde{M}}\right] .
$$

The first order terms are

$$
\begin{aligned}
\delta\left(\alpha \bar{\mu}_{2}\right) & =-\mu_{2} \frac{\widetilde{M}_{2}}{\widetilde{M}} \frac{\widetilde{\mu}_{3} \widetilde{M}_{4}+\mu_{3}^{*} \widetilde{M}_{3}}{\bar{\mu}_{3} \widetilde{M}}, \\
\delta \bar{\mu}_{1} & =\widetilde{\mu}_{1} \frac{\mu_{2}^{*}}{\bar{\mu}_{2}} \frac{\mu_{3}}{\bar{\mu}_{3}^{2}} \frac{\widetilde{M}_{2}}{\widetilde{M}_{3}} \frac{\widetilde{M}_{3}-\mu_{3}^{*} \widetilde{M}_{4}}{\bar{\mu}_{3} \widetilde{M}},
\end{aligned}
$$

\footnotetext{
${ }^{2}$ We can exploit the phase transformation $P_{3 L}(2.9)$ to absorb the phases of $\bar{\mu}_{1}, \alpha$ and make all elements of the CKM matrix real showing explicitly that the CP violation disappears.
} 
and the Jarlskog invariant reads

$$
J_{q}=-\frac{\widetilde{\mu}_{1}^{2} \widetilde{\mu}_{2}^{2} \widetilde{\mu}_{3}^{2}}{\Delta \mathscr{M}_{d}^{2}} \frac{\widetilde{M}_{2} \widetilde{M}_{3}}{\widetilde{M}^{2}}\left[\left(1-\frac{\widetilde{M}_{4}^{2}}{\widetilde{M}^{2}}\right) \operatorname{Im} \frac{\mu_{3} \mu_{2}^{*}}{\widetilde{\mu}_{3} \widetilde{\mu}_{2}}+\frac{\widetilde{M}_{3} \widetilde{M}_{4}}{\widetilde{M}^{2}} \frac{\left|\mu_{3}\right|^{2}}{\widetilde{\mu}_{3}^{2}} \operatorname{Im} \frac{\mu_{2}}{\widetilde{\mu}_{2}}\right] .
$$

We see that $J_{q}$ vanishes if either $\mu_{2}$ or $\mu_{3}$ vanish, so two complex quantities are needed to obtain $\mathrm{CP}$ violation at low energies.

It is instructive to calculate $H_{d}^{\text {eff }}$ also from the matrix $\widehat{m}$, Eq. (2.7). Here we notice that the off-diagonal elements of such matrix are relatively simple since we can exploit the unitarity of the matrix $V_{4}$, which gives $\sum_{k=1}^{3}\left(V_{4}\right)_{i k}\left(V_{4}\right)_{j k}^{*}=\delta_{i j}-\left(V_{4}\right)_{i 4}\left(V_{4}\right)_{j 4}^{*}$. So we have for $i \neq j$

$$
\left(H_{d}^{\mathrm{eff}}\right)^{i j}=\widetilde{\mu}_{i} \widetilde{\mu}_{j}\left(1-a_{i} a_{j}^{*}\right), \quad a_{i} \equiv \frac{\widetilde{\mu}_{i} \widetilde{M}_{4}+\mu_{i} \widetilde{M}_{i}}{\widetilde{\mu}_{i} \widetilde{M}}
$$

from which we get the simple expression

$$
\operatorname{Im}\left[\left(H_{d}^{\mathrm{eff}}\right)^{12}\left(H_{d}^{\mathrm{eff}}\right)^{23}\left(H_{d}^{\mathrm{eff}}\right)^{31}\right]=\widetilde{\mu}_{1}^{2} \widetilde{\mu}_{2}^{2} \widetilde{\mu}_{3}^{2} \sum_{\text {cycl. perm } i j k}\left(1+\left|a_{i}\right|^{2}\right) \operatorname{Im}\left(a_{j}^{*} a_{k}\right) .
$$

In the limit of vanishing $\mu_{i}$, we see that $a_{i}=\widetilde{M}_{4} / \widetilde{M}$; thus for $\mu_{1}=\mu_{2}=0$, the expression simplifies to

$$
\operatorname{Im}\left[\left(H_{d}^{\mathrm{eff}}\right)^{12}\left(H_{d}^{\mathrm{eff}}\right)^{23}\left(H_{d}^{\mathrm{eff}}\right)^{31}\right]=\widetilde{\mu}_{1}^{2} \widetilde{\mu}_{2}^{2} \widetilde{\mu}_{3}^{2}\left(1+\frac{\widetilde{M}_{4}^{2}}{\widetilde{M}^{2}}\right) \operatorname{Im}\left[\mu_{3}^{*} \frac{\widetilde{M}_{4}}{\widetilde{M}}+\mu_{3} \frac{\widetilde{M}_{4}}{\widetilde{M}}\right]=0
$$

For $\mu_{1}=0$ but $\mu_{2} \neq 0$, we then obtain

$$
\begin{array}{r}
\operatorname{Im}\left[\left(H_{d}^{\text {eff }}\right)^{12}\left(H_{d}^{\text {eff }}\right)^{23}\left(H_{d}^{\text {eff }}\right)^{31}\right]=\widetilde{\mu}_{1}^{2} \widetilde{\mu}_{2}^{2} \widetilde{\mu}_{3}^{2} \frac{\widetilde{M}_{2} \widetilde{M}_{3}}{\widetilde{M}^{2}}\left[\left(1-\frac{\widetilde{M}_{4}^{2}}{\widetilde{M}^{2}}\right) \operatorname{Im}\left(\frac{\mu_{3} \mu_{2}^{*}}{\widetilde{\mu}_{3} \widetilde{\mu}_{2}}\right)\right. \\
\left.+\frac{\widetilde{M_{3}} \widetilde{M}_{4}}{\widetilde{M}^{2}} \frac{\left|\mu_{3}\right|^{2}}{\widetilde{\mu}_{3}^{2}} \operatorname{Im}\left(\frac{\mu_{2}}{\widetilde{\mu}_{2}}\right)-\frac{\widetilde{M}_{2} \widetilde{M}_{4}}{\widetilde{M}^{2}} \frac{\left|\mu_{2}\right|^{2}}{\widetilde{\mu}_{2}^{2}} \operatorname{Im}\left(\frac{\mu_{3}}{\widetilde{\mu}_{3}}\right)\right] .
\end{array}
$$

The complete expression for $J_{q}$ is displayed in Eq. (A.19); the dominant terms are exactly those given in Eq. (3.9).

For degenerate heavy masses $\widetilde{M}$, the result simplifies to

$$
J_{q}=\frac{1}{16} \frac{\widetilde{\mu}_{1}^{2} \widetilde{\mu}_{2}}{\Delta \mathscr{M}_{d}^{2}}\left(3 \widetilde{\mu}_{3} \operatorname{Im}\left(\mu_{3} \mu_{2}^{*}\right)+\left|\mu_{3}\right|^{2} \operatorname{Im}\left(\mu_{2}\right)\right) .
$$

Note that the numerical factor, $\frac{1}{16}$, is minimal for degenerate $\widetilde{M}$. Due to the hierarchy of the down quarks, $\Delta \mathscr{M}_{d}^{2} \simeq m_{s}^{2} m_{b}^{4} \simeq \bar{\mu}_{2}^{2} \bar{\mu}_{3}^{4}$. So we finally obtain, substituting the order of magnitude of the parameters, with $\widetilde{\mu}_{3} \simeq\left|\mu_{3}\right|$,

$$
J_{q} \simeq V_{u s} \frac{m_{d} m_{s}}{m_{b}^{2}} \frac{1}{4 \sqrt{2}}\left(3 \sin \left(\theta_{3}-\theta_{2}\right)+\sin \theta_{2}\right) \simeq 10^{-5}\left(3 \sin \left(\theta_{3}-\theta_{2}\right)+\sin \theta_{2}\right) .
$$


This is the right order of magnitude; the current experimental value is $J_{q}=3 \times 10^{-5}$ [29]. From Eq. (3.14) we can conclude that a single complex parameter, with the other two vanishing, is not enough to have low-energy $\mathrm{CP}$ violation in the quark sector and that the CKM phase is a combination of the high-energy phases $\theta_{i}$ weighted by mass hierarchies. Moreover, maximal phases seem to be needed to give the large low-energy phase observed.

\section{4. $\mathrm{CP}$ violation in the leptonic sector}

The charged lepton and Dirac neutrino mass matrices can be transformed like the down quark mass matrix. The heavy state is an $\mathrm{SU}(2)_{L}$ doublet, so $V_{4}$ singles out the same state for charged leptons and neutrinos.

The effective $3 \times 3$-matrices read (cf. Eq. (2.7))

$$
\widehat{m}^{e}=\left(\begin{array}{l}
\mu_{1}\left(V_{4}\right)_{1 j}+\widetilde{\mu}_{1}\left(V_{4}\right)_{4 j} \\
\mu_{2}\left(V_{4}\right)_{2 j}+\widetilde{\mu}_{2}\left(V_{4}\right)_{4 j} \\
\mu_{3}\left(V_{4}\right)_{3 j}+\widetilde{\mu}_{3}\left(V_{4}\right)_{4 j}
\end{array}\right), \quad \widehat{m}^{D}=\left(\begin{array}{c}
\rho_{1}\left(V_{4}\right)_{1 j}+\widetilde{\rho}_{1}\left(V_{4}\right)_{4 j} \\
\rho_{2}\left(V_{4}\right)_{2 j}+\widetilde{\rho}_{2}\left(V_{4}\right)_{4 j} \\
\rho_{3}\left(V_{4}\right)_{3 j}+\widetilde{\rho}_{3}\left(V_{4}\right)_{4 j}
\end{array}\right) .
$$

Within our model we assume the hierarchical patterns of $\mu_{i}$ and $\rho_{i}$ as well as $\widetilde{\mu}_{i}$ and $\widetilde{\rho}_{i}(i=1 . .3)$ to be the same as for down quarks. The precise values, however, can be different since they originate from different Yukawa couplings, see Eqs. (2.30). Again, we choose the couplings between the brane states, $\mu_{i}$ and $\rho_{i}$, complex.

Although some of the charged lepton and down quark parameters, namely $\mu_{1}$ and $\mu_{3}$, are related by GUT symmetries, the corresponding phases after the redefinition leading to Eq. (2.6) are completely uncorrelated. Thus, there is no direct relation between the $\mathrm{CP}$ violation in the leptonic and in the hadronic observables, even though, barring cancellations, we expect the leptonic $\mathrm{CP}$ violation to be large as well. Furthermore, we will see that different combinations of the phases determine the experimental observables. Thus even if there were relations between the phases in the quark and lepton sector, these would not be observable. Some correlations, however, could survive between charged and neutral leptons. As in the quark sector, we expect similar suppression for the $\mathrm{CP}$ violation due to the specific mass texture in our model.

The discussion of the charged lepton masses closely follows the discussion of the down quarks in the previous section. The parameters are chosen such that they match the observed hierarchy, as described in Appendix A.1. The light neutrino masses, however, result from the seesaw mechanism, since we have heavy Majorana masses for the right-handed neutrinos. This Majorana matrix is diagonal, but can have complex entries (cf. Eq. (2.3a)),

$$
m^{N}=\left(\begin{array}{ccc}
M_{1} e^{2 i \phi_{1}} & 0 & 0 \\
0 & M_{2} e^{2 i \phi_{2}} & 0 \\
0 & 0 & M_{3} e^{2 i \phi_{3}}
\end{array}\right)=e^{2 i \phi_{3}}\left(\begin{array}{ccc}
M_{1} e^{2 i \Delta \phi_{13}} & 0 & 0 \\
0 & M_{2} e^{2 i \Delta \phi_{23}} & 0 \\
0 & 0 & M_{3}
\end{array}\right),
$$


where $\Delta \phi_{i j}=\phi_{i}-\phi_{j}$. Altogether, we have nine independent phases in the lepton sector; in the limit of small $\mu_{1}$ and $\rho_{1}$, they reduce to seven. Since neutrinos are Majorana, we have less freedom in the phase reshuffling. However, except for electroweak breaking effects in $U_{4}$, the heavy state is effectively an $\mathrm{SU}(2)$-doublet of Dirac fermions. This allows us to absorb some phases in the Dirac mass matrix and reduce the system to three generations for both charged and neutral leptons at the same time. In the following, we will neglect any effect of this heavy fourth generation doublet and concentrate on the three light generations including the right-handed neutrinos. We expect this approximation to be valid as long as $\widetilde{M} \sim M_{\mathrm{GUT}}$ is much larger than the Majorana masses $M_{i}[16]$.

\subsection{Seesaw Mechanism and Effective Mass Matrix}

In the case of the leptons, neither $\widehat{m}^{e}$ nor $\widehat{m}^{D}$ is diagonal and therefore we will change the basis in order to simplify the discussion of the CP violation. Luckily, the large rotations of type $\widehat{V}_{3}$, which bring the Dirac matrices into triangular form, are similar for charged leptons and neutrinos, thanks to the same hierarchical structure.

To distinguish the flavour of the light neutrinos, we first act on the neutrino Dirac mass matrix with exactly the same $\widehat{V}_{3}$ that transforms the charged lepton mass matrix into the upper triangular form, see Eq. (2.8), and obtain

$$
\bar{m}^{D}=\left(\begin{array}{ccc}
A \bar{\rho}_{1} & D \bar{\rho}_{1} & \bar{\rho}_{1} \\
B \bar{\rho}_{2} & E \bar{\rho}_{2} & \bar{\rho}_{2} \\
C \bar{\rho}_{3} & F \bar{\rho}_{3} & \bar{\rho}_{3}
\end{array}\right)
$$

At this stage the charged lepton mass matrix is not yet diagonal, but not very far from it: the complete diagonalisation can be obtained by applying another nearly diagonal rotation matrix on the right, corresponding to the mismatch between $V_{3}$ and $\widehat{V}_{3}$, and a CKM-like rotation $U_{3}$ on the left as described in Appendix A. Note that such a transformation from the left, as $U_{4}$, in this case acts on the right-handed fields and leaves both $H=\bar{m}^{\dagger} \bar{m}$ and the light neutrino Majorana mass matrix,

$$
m_{\mathrm{eff}}^{\nu}=-\left(m^{D}\right)^{\top}\left(m^{N}\right)^{-1} m^{D}
$$

unchanged. In fact $U_{4}$ acts in very good approximation as the unity matrix on $m^{N}$ up to terms $\mathcal{O}\left(v^{2} / \widetilde{M}^{2}\right)$, while $U_{3}$ just cancels out.

So apart for the small rotation on the right needed to diagonalise $H$, which affects the CP violation in the neutrino oscillation only weakly (see Section 4.3), the neutrino masses and mixings can be obtained from Eq. (4.4), in the form

$$
m_{\text {eff }}^{\nu}=-\left(\begin{array}{ccc}
C^{2} \varrho_{3}+B^{2} \varrho_{2}+A^{2} \varrho_{1} & C F \varrho_{3}+B E \varrho_{2}+A D \varrho_{1} C \varrho_{3}+B \varrho_{2}+A \varrho_{1} \\
C F \varrho_{3}+B E \varrho_{2}+A D \varrho_{1} & F^{2} \varrho_{3}+E^{2} \varrho_{2}+D^{2} \varrho_{1} & F \varrho_{3}+E \varrho_{2}+D \varrho_{1} \\
C \varrho_{3}+B \varrho_{2}+A \varrho_{1} & F \varrho_{3}+E \varrho_{2}+D \varrho_{1} & \varrho_{3}+\varrho_{2}+\varrho_{1}
\end{array}\right),
$$


where $\varrho_{i}=e^{-2 i \phi_{i}} \bar{\rho}_{i}^{2} / M_{i}$. Note that the determinant of the (23)-submatrix of $m_{\mathrm{eff}}^{\nu}$ is not of order $\varrho_{3}^{2}$; instead it reads $\varrho_{3} \varrho_{2}(F-E)^{2}+\varrho_{3} \varrho_{1}(F-D)^{2}+\varrho_{2} \varrho_{1}(E-D)^{2}$, allowing a large solar mixing angle [30].

The leading part of the light neutrino mass matrix (4.5) is obtained in the limit $\mu_{1}, \rho_{1} \rightarrow 0$. From the general expressions (A.24) one obtains

$$
\begin{aligned}
& \bar{\rho}_{1}=\widetilde{\rho}_{1} \frac{1}{\bar{\mu}_{3}} \frac{1}{\widetilde{M}^{2}}\left[\widetilde{\mu}_{3} \widetilde{M}_{123}^{2}-\mu_{3}^{*} \widetilde{M}_{3} \widetilde{M}_{4}\right] \\
& \bar{\rho}_{2}=\frac{1}{\bar{\mu}_{3}} \frac{1}{\widetilde{M}^{2}}\left\{\widetilde{\rho}_{2}\left[\widetilde{\mu}_{3} \widetilde{M}_{123}^{2}-\mu_{3}^{*} \widetilde{M}_{3} \widetilde{M}_{4}\right]-\rho_{2} \widetilde{M}_{2}\left[\widetilde{\mu}_{3} \widetilde{M}_{4}+\mu_{3}^{*} \widetilde{M}_{3}\right]\right\} \\
& \bar{\rho}_{3}=\frac{1}{\bar{\mu}_{3}} \frac{1}{\widetilde{M}^{2}}\left\{\widetilde{\rho}_{3}\left[\widetilde{\mu}_{3} \widetilde{M}_{123}^{2}-\mu_{3}^{*} \widetilde{M_{3}} \widetilde{M}_{4}\right]-\rho_{3}\left[\widetilde{\mu}_{3} \widetilde{M}_{3} \widetilde{M}_{4}-\mu_{3}^{*} \widetilde{M}_{124}^{2}\right]\right\}
\end{aligned}
$$

where we have introduced $\widetilde{M}_{\alpha \beta \gamma}=\sqrt{\widetilde{M}_{\alpha}^{2}+\widetilde{M}_{\beta}^{2}+\widetilde{M}_{\gamma}^{2}}$

In our model, the Dirac neutrino mass matrix has a hierarchical structure similar to the one of down quarks and charged leptons. The three smallest elements, however, have a considerable uncertainty. Since $m_{e} \neq m_{d}$, these elements cannot be equal for the three matrices. Inspection of $m^{d}$ suggests for $\widetilde{\rho}_{1}$ the range between $m_{d}$ and $m_{s} V_{u s}$; the difference is a factor $\mathcal{O}(1)$. In the following we shall consider the case of small $\widetilde{\rho}_{1}$. For large $\widetilde{\rho}_{1}$ so that $\left|\varrho_{1}\right|>\left|\varrho_{3}\right|$, in the following discussion we should interchange $\bar{\rho}_{3}, \varrho_{3}$ with $\bar{\rho}_{1}, \varrho_{1}$ and consider it as the dominant scale.

We here assume $\bar{\rho}_{3}: \bar{\rho}_{2}: \bar{\rho}_{1} \sim \widetilde{\rho}_{3}: \widetilde{\rho}_{2}: \widetilde{\rho}_{1} \sim m_{b}: m_{s}: m_{d}$, which yields [13]

$$
\frac{\left|\varrho_{2}\right|}{\left|\varrho_{3}\right|} \sim \frac{\bar{\rho}_{2}^{2}}{\bar{\rho}_{3}^{2}} \frac{M_{3}}{M_{2}} \sim \frac{m_{s}^{2}}{m_{b}^{2}} \frac{m_{t}}{m_{c}} \sim 0.2, \quad \frac{\left|\varrho_{1}\right|}{\left|\varrho_{3}\right|} \sim \frac{\bar{\rho}_{1}^{2}}{\bar{\rho}_{3}^{2}} \frac{M_{3}}{M_{1}} \sim \frac{m_{d}^{2}}{m_{b}^{2}} \frac{m_{t}}{m_{u}} \sim 0.2
$$

such that $\varrho_{1} \sim \varrho_{2}<\varrho_{3}$. Hence, in this model, the weak hierarchy in the neutrino sector can be traced back to the nearly perfect compensation between down and up quark hierarchies.

The relation $\varrho_{1} \sim \varrho_{2}$ implies for the two small neutrino masses $\left|m_{1}\right| \sim\left|m_{2}\right|$ barring cancellations or small parameters. As computed in Appendix $\mathrm{A}$, the masses at leading order assuming $\varrho_{3}$ to dominate are given by

$$
\begin{aligned}
m_{3} & =-\varrho_{3}\left(1+|F|^{2}+|C|^{2}\right) \\
\left|m_{2} m_{1}\right| & =\left|\varrho_{2} \varrho_{1}\right| \frac{|(F-E)(A-B)+(D-E)(B-C)|^{2}}{1+|F|^{2}+|C|^{2}} .
\end{aligned}
$$

The light neutrino mass spectrum has normal hierarchy, and the ratio $m_{2}^{2} / m_{3}^{2}$ can be identified with $\Delta m_{\mathrm{sol}}^{2} / \Delta m_{\mathrm{atm}}^{2}$, which is indeed consistent with observations within the theoretical uncertainties. 
The coefficients $A \ldots F$ of the neutrino mass matrix $m_{\text {eff }}^{\nu}$ become in the limit $\mu_{1}, \rho_{1} \rightarrow 0$

$$
\begin{aligned}
& A=-\frac{\widetilde{\rho}_{1}}{\bar{\rho}_{1}} \frac{\mu_{2}}{\bar{\mu}_{2}} \frac{\mu_{3}}{\bar{\mu}_{3}} \frac{\widetilde{M_{1}}}{\widetilde{M}}, \\
& B=\frac{\rho_{2} \widetilde{\mu}_{2}-\widetilde{\rho}_{2} \mu_{2}}{\bar{\rho}_{2} \bar{\mu}_{2}} \frac{\mu_{3}}{\bar{\mu}_{3}} \frac{\widetilde{M_{1}}}{\widetilde{M}}, \\
& C=\frac{\widetilde{\mu}_{3} \rho_{3}-\mu_{3} \widetilde{\rho}_{3}}{\bar{\rho}_{3} \bar{\mu}_{3}} \frac{\mu_{2}}{\bar{\mu}_{2}} \frac{\widetilde{M_{1}}}{\widetilde{M}}, \\
& D=\frac{\widetilde{\rho}_{1}}{\bar{\rho}_{1}} \frac{1}{\bar{\mu}_{2}} \frac{1}{\bar{\mu}_{3}^{2}} \frac{1}{\widetilde{M}^{2}}\left[\widetilde{\mu}_{2}\left|\mu_{3}\right|^{2} \widetilde{M}_{12}^{2}+\mu_{2}^{*} \mu_{3} \widetilde{M}_{2}\left(\widetilde{\mu}_{3} \widetilde{M}_{3}-\mu_{3}^{*} \widetilde{M}_{4}\right)\right] \\
& F=\frac{1}{\bar{\rho}_{3}} \frac{1}{\bar{\mu}_{2}} \frac{1}{\bar{\mu}_{3}^{2}} \frac{1}{\widetilde{\rho}_{2}} \frac{1}{\bar{\mu}_{2}} \frac{1}{\bar{\mu}_{3}} \frac{1}{\widetilde{M}^{2}}\left[\widetilde{\mu}_{3} \mu_{3}^{*} \widetilde{M}_{2}-\widetilde{M}_{3}+\rho_{3}\left(\widetilde{\mu}_{3}\right)\left[\widetilde{\mu}_{2} \widetilde{M}_{3}^{*} \widetilde{M}_{12}^{2}+\mu_{3}^{*} \widetilde{M}_{3} \widetilde{M}_{4}\right)\right]
\end{aligned}
$$

Note that $B, C, F$ vanish in the limiting case of equal hierarchy in the neutrino and charged lepton Dirac mass matrix, i.e., for $\rho_{i} / \widetilde{\rho}_{i}=\mu_{i} / \widetilde{\mu}_{i}$, and $A$ is in this case proportional to $\gamma \bar{\mu}_{1}$. In fact, if the neutrino and charged lepton vectors are perfectly aligned in flavour space the neutrino Dirac matrix becomes triangular at the same time as the charged lepton one and we cannot reproduce large neutrino mixing. There is though no reason to expect such alignment since the parameters $\widetilde{\rho}_{i}, \widetilde{\mu}_{i}$ are not related by any GUT relation, as can be seen in Eq. (2.30). So the large neutrino mixing angles are not generated simply by the large LH rotation contained in the charged lepton's $\widehat{V}_{3}$, but from its misalignment with the neutrinos.

Using the relations between $\widetilde{\rho}_{i}, \bar{\rho}_{i}$ and $\rho_{i}$, and $\widetilde{\mu}_{i}, \bar{\mu}_{i}$ and $\mu_{i}$ due to the hierarchical structure of the mass matrices in our model, one obtains the simple expressions,

$$
A \sim C \sim \frac{\mu_{2}}{\bar{\mu}_{2}}, \quad B \sim \frac{\rho_{2}}{\bar{\rho}_{2}}-\frac{\mu_{2}}{\bar{\mu}_{2}}, \quad D \sim E \sim F \sim 1 .
$$

The mixing angles are computed in Appendix A.2; in the case the parameters $A, C$ are small, they are given by

$$
\begin{aligned}
\tan \theta_{23} & \simeq|F|, \\
\tan \theta_{12} & \sim \frac{|B|}{|E-F|} \sqrt{1+|F|^{2}}, \\
\sin \theta_{13} & \sim \frac{C}{\sqrt{1+|F|^{2}}}+\frac{B(E F+1)}{\left(1+|F|^{2}\right)^{3 / 2}} \frac{\left|\varrho_{2}\right|}{\left|\varrho_{3}\right|} .
\end{aligned}
$$

The atmospheric mixing angle $\theta_{23}$ is naturally large; the current best fit $[29,31]$ restricts the parameter $F$ as $0.7 \lesssim|F| \lesssim 1.4$ to have it maximal. Note that $F \geq 0.7$ can naturally be obtained even for $\left|\rho_{3}\right| / \widetilde{\rho}_{3} \sim\left|\mu_{3}\right| / \widetilde{\mu}_{3}$, as discussed in Appendix A.2. 
For $\left(\mu_{2} / \bar{\mu}_{2}\right)_{e} \sim\left(\mu_{2} / \bar{\mu}_{2}\right)_{d} \sim 0.1$ one then obtains $|C| \sim 0.1$ and a value for $\theta_{13}$ close to the current upper bound. In this case though, $\tilde{\mu}_{1}^{e}$ has to be suppressed with respect to the down quark case in order to give a consistently small $m_{e}$. The large solar mixing $\theta_{12}$ can then be achieved for $B \sim 0.1-1$ with moderate tuning of $E-F$.

Another possibility is that a very small $\mu_{2}$ is called for to explain the smallness of the electron mass. In this case, we have naturally $|A|,|C| \sim 0.01$ and the reactor angle is dominated by the second term in Eq. (4.11). Then the angles $\theta_{12}$ and $\theta_{13}$ depend on the same parameter $B$, but for the second one there is a suppression by $\varrho_{2} / \varrho_{3}$. So in the case of hierarchical $\varrho_{i}$, both a large and small angle can be explained even with relatively large $B$. Such value for $B$ is not unnatural, even for small $\mu_{2}$, if we accept $\rho_{2}>\left(\mu_{2}\right)_{e}$. In this case we have $\sin \theta_{13} \lesssim 0.1$ correlated with the mass eigenvalues $m_{1} \lesssim m_{2} \lesssim m_{3}$. Note that in general, if all parameters $A, B$, and $C$ are smaller than one, we obtain the prediction $m_{1}<m_{2}$, while for $B \sim 1$ the two lowest eigenvalues are nearly degenerate.

The largest of the heavy neutrino masses is given by $M_{3} \sim m_{t}^{2} / \sqrt{\Delta m_{\mathrm{atm}}^{2}} \sim$ $10^{15} \mathrm{GeV}$. For the lightest heavy Majorana state the model provides the rough estimate $M_{1} \sim M_{3} m_{u} / m_{t} \sim 10^{10} \mathrm{GeV}$.

\subsection{Neutrinoless Double Beta Decay $(0 \nu \beta \beta)$}

The simultaneous decay of two neutrons may result in neutrinoless double beta decay, e.g., ${ }^{78} \mathrm{Ge} \rightarrow{ }^{76} \mathrm{Se}+2 e$. This process is currently most promising to prove the Majorana nature of neutrinos. The decay width can be expressed as

$$
\Gamma=G\left|\mathcal{M}^{2}\right|\left|m_{e e}\right|^{2},
$$

where $G$ is a phase space factor, $\mathcal{M}$ the nuclear $0 \nu \beta \beta$ matrix element, and $m_{e e}$ is the (11)-element of the light neutrino mass matrix.

Since the electron mass is very small, the charged lepton mass matrix in triangular form has nearly a vanishing first row. Then the left-handed electron is already singled out; the remaining rotation mostly affects the (23)-block. Therefore we can already make an estimate of $m_{e e}$ from the effective neutrino Majorana matrix, $m_{\text {eff }}^{\nu}$. From Eq. (4.5), we read off

$$
\left|m_{e e}\right|=\left|C^{2} \varrho_{3}+B^{2} \varrho_{2}+A^{2} \varrho_{1}\right|,
$$

where the last term can be negleted. This result has the same form as the standard formula in the case of hierarchical neutrinos [32],

$$
\left|m_{e e}\right|=\left|\sqrt{\Delta m_{\mathrm{atm}}^{2}} \sin ^{2} \theta_{13} e^{i\left(\xi_{3}-\xi_{2}\right)}+\sqrt{\Delta m_{\mathrm{sol}}^{2}} \sin ^{2} \theta_{12} \cos ^{2} \theta_{13}\right|,
$$

where $\xi_{3}$ and $\xi_{2}$ are the two Majorana phases in the conventional parametrization of neutrino mass matrix (A.34). 
We can estimate the size of $\left|m_{e e}\right|$ in our model using

$$
\left|\rho_{3}\right| \simeq \widetilde{\rho}_{3}, \quad\left|\mu_{3}\right| \simeq \widetilde{\mu}_{3}, \quad \frac{\rho_{2}}{\bar{\rho}_{2}} \sim 1, \quad\left|\varrho_{3}\right| \simeq \sqrt{\Delta m_{\mathrm{atm}}^{2}}, \quad\left|\varrho_{2}\right| \simeq \sqrt{\Delta m_{\mathrm{sol}}^{2}}
$$

which gives

$$
\left|m_{e e}\right| \sim\left|\frac{\mu_{2}^{2}}{\bar{\mu}_{2}^{2}} \sqrt{\Delta m_{\mathrm{atm}}^{2}} e^{2 i\left(\phi_{2}-\phi_{3}\right)}+\frac{\rho_{2}^{2}}{\bar{\rho}_{2}^{2}} \sqrt{\Delta m_{\mathrm{sol}}^{2}}\right| .
$$

Clearly, the last term dominates, yielding the familiar result for hierarchical neutrinos $\left|m_{e e}\right| \lesssim \sqrt{\Delta m_{\text {sol }}^{2}} \sim 0.01 \mathrm{eV}$ if $\mu_{2} / \widetilde{\mu}_{2} \ll \rho_{2} / \widetilde{\rho}_{2}$.

\subsection{CP Violation in Neutrino Oscillations}

Leptonic CP violation at low energies can be detected via neutrino oscillations, which are sensitive to the Dirac phase of the light neutrino mass matrix. For a diagonal charged lepton mass matrix, the strength of Dirac-type CP violation is obtained from the invariant [27]

$$
\operatorname{tr}\left[h^{\nu}, h^{e}\right]^{3}=6 i \Delta \mathscr{M}_{e}^{2} \operatorname{Im}\left[\left(h^{\nu}\right)^{12}\left(h^{\nu}\right)^{23}\left(h^{\nu}\right)^{31}\right]
$$

where $h^{\nu}=\left(m^{\nu}\right)^{\dagger} m^{\nu}$ and $\Delta \mathscr{M}_{e}^{2}$ is the product of the mass squared differences of the charged leptons, cf. Eq. (3.2). This quantity is connected to the leptonic equivalent of the Jarlskog invariant through

$$
J_{\ell}=-\frac{1}{\mathscr{M}_{\nu}^{2}} \operatorname{Im}\left[\left(h^{\nu}\right)^{12}\left(h^{\nu}\right)^{23}\left(h^{\nu}\right)^{31}\right],
$$

where

$$
\Delta \mathscr{M}^{2}=\left(m_{3}^{2}-m_{2}^{2}\right)\left(m_{3}^{2}-m_{1}^{2}\right)\left(m_{2}^{2}-m_{1}^{2}\right)=\delta m_{\mathrm{sol}}^{2} \delta m_{\mathrm{atm}}^{4} \sim\left|\varrho_{2}\right|^{2}\left|\varrho_{3}\right|^{4}
$$

is now the product of the light neutrino mass squared differences. In the standard parametrisation given in Eq. (A.34),

$$
J_{\ell}=\operatorname{Im}\left[\left(V_{\nu}\right)_{11}\left(V_{\nu}\right)_{22}\left(V_{\nu}\right)_{12}^{*}\left(V_{\nu}\right)_{21}^{*}\right]=\frac{1}{8} \cos \theta_{13} \sin 2 \theta_{13} \sin 2 \theta_{12} \sin 2 \theta_{23} \sin \delta,
$$

where $\delta$ is the CP violating Dirac phase in the SM with massive neutrinos.

The expressions (4.18) and (4.20) assume that the charged lepton mass matrix is diagonal. In our case, this matrix is nearly diagonal after the $\widehat{V}_{3}$ rotation, as the electron mass is very small; in fact, the remaining rotation $V_{3}^{\prime}$ deviates from a unit matrix only in the 23 sector and at order $\mathcal{O}\left(m_{\mu}^{2} / m_{\tau}^{2}\right) \ll 1$ (see Eq. (A.13)). Therefore up to corrections of this order, we can use Eq. (4.18) with the effective neutrino mass matrix $m_{\text {eff }}^{\nu}$ given in Eq. (4.5), i.e.,

$$
J_{\ell}=-\frac{1}{\mathscr{M}_{\nu}^{2}} \operatorname{Im}\left[\left(h_{\text {eff }}^{\nu}\right)^{12}\left(h_{\text {eff }}^{\nu}\right)^{23}\left(h_{\text {eff }}^{\nu}\right)^{31}\right],
$$


with now $h_{\text {eff }}^{\nu}=\left(m_{\text {eff }}^{\nu}\right)^{\dagger} m_{\text {eff. }}^{\nu}$ We compute the first few terms and obtain

$$
\begin{aligned}
& \left(h_{\mathrm{eff}}^{\nu}\right)^{12}=\frac{C^{*} F\left|m_{3}\right|^{2}}{1+|F|^{2}+|C|^{2}}+C^{*} E\left(1+F^{*} E+C^{*} B\right) \varrho_{2} \varrho_{3}^{*}+B^{*} F\left(1+F^{*} E+C^{*} B\right)^{*} \varrho_{2}^{*} \varrho_{3}, \\
& \left(h_{\mathrm{eff}}^{\nu}\right)^{23}=\frac{F^{*}\left|m_{3}\right|^{2}}{1+|F|^{2}+|C|^{2}}+F^{*}\left(1+F^{*} E+C^{*} B\right) \varrho_{2} \varrho_{3}^{*}+E^{*}\left(1+F^{*} E+C^{*} B\right)^{*} \varrho_{2}^{*} \varrho_{3}, \\
& \left(h_{\mathrm{eff}}^{\nu}\right)^{31}=\frac{C\left|m_{3}\right|^{2}}{1+|F|^{2}+|C|^{2}}+B\left(1+F^{*} E+C^{*} B\right) \varrho_{2} \varrho_{3}^{*}+C\left(1+F^{*} E+C^{*} B\right)^{*} \varrho_{2}^{*} \varrho_{3} .
\end{aligned}
$$

The leading contribution in the cyclic product of $h_{\text {eff }}^{\nu}$, which is $\propto\left|m_{3}\right|^{6}$, is real and does not contribute to $J_{\ell}$; that is to be expected since it corresponds to the limit of two massless neutrinos where no physical Dirac phase can be defined. In general the first non-trivial terms are of order $\left|\varrho_{3}\right|^{4}\left|\varrho_{2}\right|^{2}$, as $\Delta \mathscr{M}_{\nu}^{2}$, so that we expect $\left|J_{\ell}\right| \lesssim 1$. We obtain in fact

$$
J_{\ell} \sim \frac{\left(1+|B|^{2}+|E|^{2}\right)\left(|E|^{2}-|F|^{2}+|B|^{2}-|C|^{2}\right)}{\left(1+|F|^{2}+|C|^{2}\right)^{3}} \operatorname{Im}\left[C^{*} F(F-E)^{*}(B-C)\right] .
$$

Note that the imaginary part vanishes for $E=F$ or $B=C$, when the flavour eigenvectors are partially aligned. Furthermore, the contribution disappears for $C=0$, so it is suppressed by the small reactor angle as expected. Due to the unknown parameters $\mathcal{O}(1)$, no useful upper bound on $J_{\ell}$ can be derived in the general case, but we see that the Dirac CP phase is given by a combination of the phases of the neutrino Dirac mass coefficients $B, C, E$ and $F$, derived from the complex parameters $\mu_{3}, \mu_{2}$, $\rho_{3}, \rho_{2}$. No dependence arises from the heavy neutrino Majorana phases $\phi_{3,2}$ since they cancel out in $\left|\varrho_{3}\right|^{4}\left|\varrho_{2}\right|^{2}$.

In the limit $\mu_{2} \rightarrow 0$, where $A=C=0$, but with $B$ of order unity, the dominant contribution to $J_{\ell}$ comes from higher order terms. We can obtain it from

$$
\begin{aligned}
& \left(h_{\mathrm{eff}}^{\nu}\right)^{12}=B^{*} F\left(1+F^{*} E\right)^{*} \varrho_{2}^{*} \varrho_{3}+B^{*} E\left(1+|B|^{2}+|E|^{2}\right)\left|\varrho_{2}\right|^{2}, \\
& \left(h_{\mathrm{eff}}^{\nu}\right)^{23}=F^{*}\left|\varrho_{3}\right|^{2}\left(1+|F|^{2}\right)+F^{*}\left(1+F^{*} E\right) \varrho_{2} \varrho_{3}^{*}+E^{*}\left(1+F^{*} E\right)^{*} \varrho_{2}^{*} \varrho_{3}, \\
& \left(h_{\mathrm{eff}}^{\nu}\right)^{31}=B\left(1+F^{*} E\right) \varrho_{2} \varrho_{3}^{*}+B\left(1+|B|^{2}+|E|^{2}\right)\left|\varrho_{2}\right|^{2} .
\end{aligned}
$$

Note that the leading term, proportional to $|B|^{2}\left|\varrho_{3}\right|^{4}\left|\varrho_{2}\right|^{2}$, is real, and in fact we did not have any $|B|^{2}$ contributions at that order above. Hence, we consider the next terms,

$$
\begin{aligned}
\left(h_{\mathrm{eff}}^{\nu}\right)^{12}\left(h_{\mathrm{eff}}^{\nu}\right)^{23}\left(h_{\mathrm{eff}}^{\nu}\right)^{31} \propto & \left(1+F^{*} E\right) F^{*}\left(\kappa_{1} E+\kappa_{2} F\right) \varrho_{2} \varrho_{3}^{*} \\
& +\left(1+F^{*} E\right)^{*} F\left(\kappa_{1} F^{*}+\kappa_{2} E^{*}\right) \varrho_{2}^{*} \varrho_{3},
\end{aligned}
$$


where we defined the real parameters

$$
\begin{aligned}
& \kappa_{1}=\left(1+|B|^{2}+|E|^{2}\right)\left(1+|F|^{2}\right), \\
& \kappa_{2}=\left|1+E^{*} F\right|^{2} .
\end{aligned}
$$

Note again that the two terms in Eq. (4.25) are exactly conjugate to each other for $E=F$ when the two heavy eigenstates are nearly aligned. In this limit $\tan \theta_{12}$ becomes maximal. Therefore, if $B$ gives the dominant contribution, the Dirac type $\mathrm{CP}$ violation is suppressed for maximal solar angle. The $\mathrm{CP}$ invariant vanishes as well if $B=0$ as the system effectively reduces to two generations and $\sin \theta_{13}=0$ (recall that we are already in the limit $A=C=0$ ). We then obtain

$$
\operatorname{Im}\left[\left(h_{\text {eff }}^{\nu}\right)^{12}\left(h_{\text {eff }}^{\nu}\right)^{23}\left(h_{\text {eff }}^{\nu}\right)^{31}\right]=|B|^{2}\left|\varrho_{2}\right|^{2}\left|\varrho_{3}\right|^{4}\left(\kappa_{1}-\kappa_{2}\right) \operatorname{Im}(\Omega)
$$

with

$$
\Omega=\left(1+E F^{*}\right) F^{*}(E-F) \frac{\varrho_{2}}{\varrho_{3}}
$$

which yields

$$
J_{\ell} \sim-|B|^{2}\left(\kappa_{1}-\kappa_{2}\right) \operatorname{Im}(\Omega) .
$$

Comparison with Eq. (4.20) shows then that in this case the standard Dirac phase $\delta$ is a complicated function of the phases of $\mu_{3}, \rho_{3}, \rho_{2}$ in the leptonic Dirac mass matrices, the difference between two of the Majorana phases $\Delta \phi_{32}$ and neutrino masses. It is suppressed by the ratio $\left|\varrho_{2}\right| /\left|\varrho_{3}\right|$, as is $\sin \theta_{13}$.

Whenever only few of the parameters in the Dirac neutrino mass matrix matter, we expect correlations between the lightest eigenvalue, the mixing angles and the maximal value for $J_{\ell}$. In Appendix A.2, we consider the simple case where $B$ dominates and the lightest eigenvalue $m_{1}$ vanishes; then all the observables are only function of $B, E, F, \varrho_{2} / \varrho_{3}$ and we show relations among them. In this specific case, even allowing for the uncertainty on the phases, upper bounds can be obtained for $\sin \theta_{13}, m_{e e}$ and $J_{\ell}$. In the more general case, subleading terms and other parameters become important and relax any such bounds.

\subsection{Leptogenesis}

The out-of-equilibrium decays of heavy Majorana neutrinos is a natural source of the cosmological matter-antimatter asymmetry [33]. In recent years this leptogenesis mechanism has been studied in great detail. The main ingredients are CP asymmetry and washout processes, which depend on neutrino masses and mixings.

It is convenient to work with a diagonal and real matrix for the right-handed neutrinos, which is obtained from $m^{N}$ by the phase transformation

$$
P_{M}=\operatorname{diag}\left(e^{-i \phi_{1}}, e^{-i \phi_{2}}, e^{-i \phi_{3}}\right) .
$$


For hierarchical heavy neutrinos the generated baryon asymmetry is dominated by decays of the lightest state $N_{1}$. In supersymmetric models the corresponding CP asymmetry is [34]

$$
\varepsilon_{1}=-\frac{3}{8 \pi} \sum_{i} \frac{\operatorname{Im}\left(\mathrm{M}_{1 i}^{2}\right)}{\mathrm{M}_{11} v_{u}^{2}} \frac{M_{1}}{M_{i}}, \quad \mathrm{M}=P_{M} \widehat{m}^{D} \widehat{m}^{D \dagger} P_{M}^{*},
$$

where the matrix elements are given, analogously to Eq. (3.10),

$$
\mathrm{M}_{i j}=e^{i \Delta \phi_{j i}} \widetilde{\rho}_{i} \widetilde{\rho}_{j}\left(1-b_{i} b_{j}^{*}\right), \quad b_{i} \equiv \frac{\widetilde{\rho}_{i} \widetilde{M}_{4}+\rho_{i} \widetilde{M}_{i}}{\widetilde{\rho}_{i} \widetilde{M}} .
$$

The terms involving one index 1 simplify for $\rho_{1}=0$ as

$$
\begin{aligned}
& \mathrm{M}_{11}=\widetilde{\rho}_{1}^{2}\left(1-\frac{\widetilde{M}_{4}^{2}}{\widetilde{M}^{2}}\right), \\
& \mathrm{M}_{1 j}=e^{i \Delta \phi_{j 1}} \widetilde{\rho}_{1} \widetilde{\rho}_{j}\left(1-\frac{\widetilde{M}_{4}}{\widetilde{M}} \frac{\widetilde{\rho}_{j} \widetilde{M}_{4}+\rho_{j}^{*} \widetilde{M}_{j}}{\widetilde{\rho}_{j} \widetilde{M}}\right) .
\end{aligned}
$$

The result then reads

$$
\varepsilon_{1} \simeq \frac{3}{8 \pi} \frac{M_{1}}{v_{u}^{2}}\left(1-\frac{\widetilde{M}_{4}^{2}}{\widetilde{M}^{2}}\right)^{-1} \sum_{j=2,3} \frac{\widetilde{\rho}_{j}^{2}}{M_{j}} \eta_{j},
$$

where

$$
\eta_{j}=-\operatorname{Im}\left[e^{i \Delta \phi_{j 1}}\left(1-\frac{\widetilde{M}_{4}}{\widetilde{M}} \frac{\widetilde{\rho}_{j} \widetilde{M}_{4}+\rho_{j}^{*} \widetilde{M}_{j}}{\widetilde{\rho}_{j} \widetilde{M}}\right)^{2}\right] .
$$

Since $\widetilde{\rho}_{2}^{2} M_{3} /\left(\widetilde{\rho}_{3}^{2} M_{2}\right) \sim 0.2$, the CP asymmetry is dominated by the intermediate state $N_{3}$, i.e., $\varepsilon_{1} \simeq 3 /(8 \pi) M_{1} \sqrt{\Delta m_{\mathrm{atm}}^{2}} / v_{u}^{2}$. In any case, the phases involved, $\Delta \phi_{13}, \Delta \phi_{12}$ and the phases of $\rho_{3}, \rho_{2}$, are completely independent of the low-energy $\mathrm{CP}$ violating phase in the quark sector and also not so directly connected to that in neutrino oscillations (even if they can contribute to it). For $M_{1} \sim 10^{10} \mathrm{GeV}$, one obtains $\varepsilon_{1} \sim 10^{-6}$, with a baryogenesis temperature $T_{B} \sim M_{1} \sim 10^{10} \mathrm{GeV}$. These are typical parameters of thermal leptogenesis $[35,36]$.

The strength of the washout processes crucially depends on the effective neutrino mass

$$
\widetilde{m}_{1}=\frac{\mathrm{M}_{11}}{M_{1}}=\frac{\widetilde{\rho}_{1}^{2}}{M_{1}}\left(1-\frac{\widetilde{M}_{4}^{2}}{\widetilde{M}^{2}}\right) \sim \varrho_{1} \lesssim 0.01 \mathrm{eV} .
$$

With the efficiency factor [37]

$$
\kappa_{f} \sim 10^{-2}\left(\frac{0.01 \mathrm{eV}}{\widetilde{m}_{1}}\right)^{1.1} \sim 10^{-2},
$$


one obtains for the baryon asymmetry

$$
\eta_{B} \sim 10^{-2} \varepsilon \kappa_{f} \sim 10^{-8} \kappa_{f} \sim 10^{-10}
$$

consistent with observation. So for successful leptogenesis we need a non vanishing $\widetilde{\rho}_{1}, \varrho_{1}$ and in particular $\varrho_{1} \sim \varrho_{2}$. In such case a zero neutrino eigenvalue is only possible due to alignment.

In the above estimate of the baryon asymmetry we have summed over the lepton flavours in the final state. In general, the CP asymmetries as well as the washout processes depend on the lepton flavour, which can lead to a considerable enhancement of the generated baryon asymmetry [38,39]. The neutrino masses $M_{1} \sim 10^{10} \mathrm{GeV}$, $\widetilde{m}_{1} \sim 0.01 \mathrm{eV}$ lie in the 'fully flavoured regime' where these effects can indeed be important [40]. Hence, depending on the $\mathrm{CP}$ violating phases the generated asymmetry may be significantly larger than the estimate (4.37).

\section{Conclusions}

We have studied in detail a specific pattern of quark and lepton mass matrices obtained from a six-dimensional GUT model compactified on an orbifold. Up quarks and right-handed neutrinos have diagonal $3 \times 3$ matrices with the same hierarchy whereas down quarks, charged leptons and Dirac neutrino mass terms are described by $4 \times 4$ matrices which have one large eigenvalue $\mathcal{O}\left(M_{\mathrm{GUT}}\right)$. The origin of this pattern are diagonal mass terms for three ordinary quark-lepton families together with large mixings $\mathcal{O}\left(M_{\mathrm{GUT}}\right)$ with a pair of $\mathrm{SU}(5)(\mathbf{5}+\overline{\mathbf{5}})$ plets. This vectorial fourth generation though is made of different split multiplets allowing for a relaxation of GUT relations. The six mass parameters of the model in the quark sector can be fixed by the up and down quark masses. This pattern of mass matrices has several remarkable features: The CKM matrix is correctly predicted and the electron mass is naturally different from the down quark mass.

The mismatch between down and up quark mass hierarchies leads, via the seesaw mechanism, to three light neutrino masses with a much milder hierarchy. Left-handed leptons and right-handed quarks have large mixings. This leads to large neutrino mixings and to small CKM mixings of the left-handed down quarks in agreement with observation.

Factors $\mathcal{O}(1)$ of the mass matrices are unknown, and the predictive power of the model is therefore limited. The neutrino mixings $\sin \theta_{23} \sim 1$ and $\sin \theta_{13} \lesssim 0.1$ are naturally accommodated. The corresponding neutrino masses are $m_{1} \lesssim m_{2} \sim$ $\sqrt{\Delta m_{\mathrm{sol}}^{2}}<m_{3} \sim \sqrt{\Delta m_{\mathrm{atm}}^{2}}$ and $\left|m_{e e}\right| \sim \sqrt{\Delta m_{\mathrm{sol}}^{2}} \lesssim 0.01 \mathrm{eV}$.

The elements of the mass matrices arise from a large number of different operators. Hence, most of the $\mathrm{CP}$ violating high-energy phases are unrelated. We find that the measured $\mathrm{CP}$ violation in the quark sector can be obtained, even if the $\mathrm{CP}$ 
invariant is suppressed by the alignment between the two lightest mass eigenstates. Due to the uncertainties of $\mathcal{O}(1)$ factors no useful upper bound on the $\mathrm{CP}$ violation in neutrino oscillations is obtained in general. Some constraints can be given in the limited case where the number of dominant parameters is reduced, as it happens if the parameters $A, C$ in the neutrino Dirac mass matrix are suppressed by the smallness of the electron mass. It is indeed intriguing that in our setting the smallness of the reactor angle can be connected to the lightness of the electron. The model is consistent with thermal leptogenesis, with a possible enhancement of the baryon asymmetry by flavour effects.

We conclude that mixings $\mathcal{O}\left(M_{\mathrm{GUT}}\right)$ of three sequential quark-lepton families with vectorial split multiplets, a pair of lepton doublets and right-handed down quarks, can account simultaneously for small quark mixings and large neutrino mixings in the charged weak current and, correspondingly, for hierarchical quark masses together with almost degenerate neutrino masses. The CP phases in the quark sector, neutrino oscillations and leptogenesis are unrelated. Quantitative predictions for the lightest neutrino mass $m_{1}$ and $\sin \theta_{13}$ require currently unknown $\mathcal{O}(1)$ factors in more specific GUT models.

\section{Acknowledgements}

We would like to thank R. Fleischer and S. Willenbrock for helpful discussions. SW thanks the SLAC Theoretical Physics Group, the DESY Theory Group, and the CERN Theory Division for hospitality during various stages of this work. LC acknowledges the support of the "Impuls- and Vernetzungsfond" of the Helmholtz Association, contract number VH-NG-006. SW is supported in part by the U. S. Department of Energy under contract No. DE-FG02-91ER40677. The work of DEC is presently supported by a CFTP-FCT UNIT 777 fellowship and through the projects POCTI/FNU/44409/2002, PDCT/FP/63914/2005, PDCT/FP/63912/2005 (Fundação para a Ciência e a Tecnologia - FCT, Portugal). 


\section{A. Mass matrices}

We will discuss here the mass eigenvalues and the mixing matrices for the low energy theory in relation to the high energy parameters.

Given a general matrix of the form as in Eq. (2.4),

$$
m=\left(\begin{array}{cccc}
\mu_{1} & 0 & 0 & \widetilde{\mu}_{1} \\
0 & \mu_{2} & 0 & \widetilde{\mu}_{2} \\
0 & 0 & \mu_{3} & \widetilde{\mu}_{3} \\
\widetilde{M_{1}} & \widetilde{M}_{2} & \widetilde{M}_{3} & \widetilde{M}_{4}
\end{array}\right),
$$

where $\mu_{i}, \widetilde{\mu}_{i}=\mathcal{O}\left(v_{1,2}\right)$ and $\widetilde{M}_{i}=\mathcal{O}\left(M_{\mathrm{GUT}}\right)$, the matrices $U_{4}$ and $V_{4}$ that single out the heavy state can be given as [14]

$$
\begin{aligned}
& U_{4} \simeq\left(\begin{array}{cccc}
1 & 0 & 0 & \frac{\mu_{1} \widetilde{M}_{1}+\widetilde{\mu}_{1} \widetilde{M}_{4}}{\widetilde{M}^{2}} \\
0 & 1 & 0 & \frac{\mu_{2} \widetilde{M}_{2}+\widetilde{\mu}_{2} \widetilde{M}_{4}}{\widetilde{M}^{2} \widetilde{M}_{4}} \\
0 & 0 & 1 & \frac{\mu_{3} \widetilde{M}_{3}+\widetilde{\mu}_{3} \widetilde{M}_{4}}{\widetilde{M}^{2}} \\
-\frac{\mu_{1} \widetilde{M}_{1}+\widetilde{\mu}_{1} \widetilde{M}_{4}}{\widetilde{M}^{2}}-\frac{\mu_{2} \widetilde{M}_{2}+\widetilde{\mu}_{2} \widetilde{M}_{4}}{\widetilde{M}^{2}}-\frac{\mu_{3} \widetilde{M}_{3}+\widetilde{\mu}_{3} \widetilde{M}_{4}}{\widetilde{M}^{2}} & 1
\end{array}\right),
\end{aligned}
$$

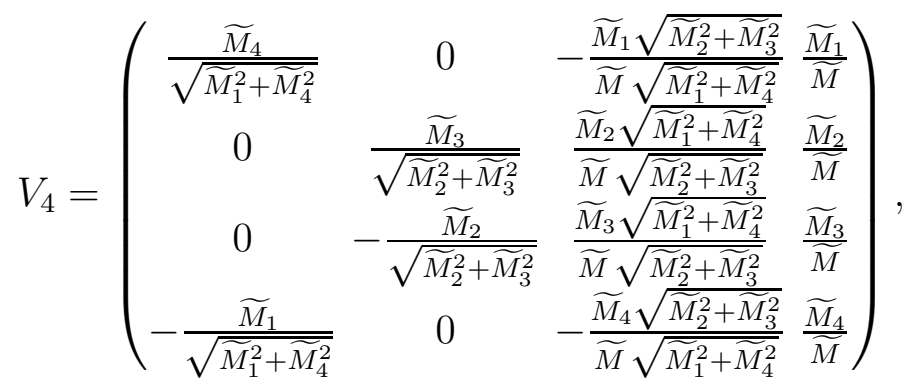

with $\widetilde{M}=\sqrt{\sum_{i} \widetilde{M}_{i}^{2}}$. In general $V_{4}$ contains large mixings, while $U_{4}$ is approximately the unity matrix, up to terms $\mathcal{O}(v / \widetilde{M})$. Next, $U_{3}$ and $V_{3}=\widehat{V}_{3} V_{3}^{\prime}$ diagonalise

$$
m^{\prime}=U_{4}^{\dagger} m V_{4}=\left(\begin{array}{cc}
\widehat{m} & 0 \\
0 & \widetilde{M}
\end{array}\right)+\mathcal{O}\left(\frac{v^{2}}{\widetilde{M^{2}}}\right),
$$

so both $U_{3}$ and $V_{3}$ have a non-trivial $3 \times 3$ part only. In the following we will use the symbols $U_{3}, V_{3}$ both for the that non-trivial upper-left corners and the full $4 \times 4$ matrices obtained adding a row and column of zeros and a diagonal 1 to those. The effective mass matrix $\widehat{m}$ can be brought into the upper triangular form by a unitary matrix $\widehat{V}_{3} \sim V_{3}$ such that

$$
\bar{m}=\widehat{m} \widehat{V}_{3}=\left(\begin{array}{ccc}
\gamma \bar{\mu}_{1} & \bar{\mu}_{1} & \beta \bar{\mu}_{1} \\
0 & \bar{\mu}_{2} & \alpha \bar{\mu}_{2} \\
0 & 0 & \bar{\mu}_{3}
\end{array}\right)
$$


With $v_{i}=\left(\widehat{m}_{i 1}, \widehat{m}_{i 2}, \widehat{m}_{i 3}\right)$, the new basis is given by

$$
\vec{e}_{3}=\frac{\vec{v}_{3}}{\left|\vec{v}_{3}\right|}, \quad \vec{e}_{2}=\frac{\vec{v}_{2}}{\left|\vec{v}_{2}\right|}-\frac{\vec{e}_{3}^{*} \cdot \vec{v}_{2}}{\vec{v}_{2}} \vec{e}_{3}, \quad \vec{e}_{1}=\vec{e}_{2} \times \vec{e}_{3} .
$$

Note that $V_{3}$ corresponds to a large angle rotation for the right-handed quark fields.

While $\bar{\mu}_{3}$ and $\bar{\mu}_{2}$ are real by construction, we have the freedom to choose any entry of the first row to be real. For concrete calculations, it is convenient to have $\gamma \bar{\mu}_{1}$ real or even use the parameters as given in the basis (A.3); however, $\gamma \bar{\mu}_{1}$ vanishes in the limit $\mu_{2} \rightarrow 0$, so for a general discussion, it is more appropriate to have $\bar{\mu}_{1}$ real. Here, we list the entries of $\bar{m}$ with $\gamma \bar{\mu}_{1}$ real in a general form,

$$
\begin{aligned}
& \bar{\mu}_{3}=\left|v_{3}\right|=\sqrt{\left|\mu_{3}\right|^{2}+\left|\widetilde{\mu}_{3}\right|^{2}-\frac{1}{M}\left|\mu_{3} \widetilde{M}_{3}+\widetilde{\mu}_{3} \widetilde{M}_{4}\right|^{2}} \\
& \alpha \bar{\mu}_{2}=\frac{\widetilde{\mu}_{2} \widetilde{\mu}_{3}^{*}}{\bar{\mu}_{3}}-\frac{\mu_{2} \widetilde{M}_{2}+\widetilde{\mu}_{2} \widetilde{M}_{4}}{\widetilde{M}} \frac{\mu_{3}^{*} \widetilde{M}_{3}+\widetilde{\mu}_{3} \widetilde{M}_{4}}{\bar{\mu}_{3} \widetilde{M}} \\
& \bar{\mu}_{2}=\sqrt{\left|\mu_{2}\right|^{2}+\left|\widetilde{\mu}_{2}\right|^{2}-\frac{1}{\widetilde{M}^{2}}\left|\mu_{2} \widetilde{M}_{2}+\widetilde{\mu}_{2} \widetilde{M}_{4}\right|^{2}-\left|\alpha \bar{\mu}_{2}\right|^{2}} ; \\
& \beta \bar{\mu}_{1}=\frac{\widetilde{\mu}_{1} \widetilde{\mu}_{3}}{\bar{\mu}_{3}}-\frac{\mu_{1} \widetilde{M}_{1}+\widetilde{\mu}_{1} \widetilde{M}_{4}}{\widetilde{M}} \frac{\mu_{3}^{*} \widetilde{M}_{3}+\widetilde{\mu}_{3} \widetilde{M}_{4}}{\bar{\mu}_{3} \widetilde{M}} \\
& \bar{\mu}_{1}=\widetilde{\mu}_{1}\left(\frac{\widetilde{\mu}_{2}}{\bar{\mu}_{2}}-\alpha^{*} \frac{\widetilde{\mu}_{3}}{\bar{\mu}_{3}}\right)-\frac{\mu_{1} \widetilde{M}_{1}+\widetilde{\mu}_{1} \widetilde{M}_{4}}{\widetilde{M}}\left[\frac{\widetilde{M}}{\widetilde{M}}\left(\frac{\widetilde{\mu}_{2}}{\bar{\mu}_{2}}-\alpha^{*} \frac{\widetilde{\mu}_{3}}{\bar{\mu}_{3}}\right)+\frac{\mu_{2}^{*}}{\bar{\mu}_{2}} \frac{\widetilde{M_{2}}}{\widetilde{M}}-\alpha^{*} \frac{\mu_{3}^{*}}{\bar{\mu}_{3}} \frac{\widetilde{M}_{3}}{\widetilde{M}}\right] \\
& \gamma \bar{\mu}_{1}=\left|\gamma \bar{\mu}_{1}\right|=\sqrt{\left|\mu_{1}\right|^{2}+\left|\widetilde{\mu}_{1}\right|^{2}-\frac{1}{\bar{M}^{2}}\left|\mu_{1} \widetilde{M}_{1}+\widetilde{\mu}_{1} \widetilde{M}_{4}\right|^{2}-\left|\bar{\mu}_{1}\right|^{2}\left(1+|\beta|^{2}\right)} .
\end{aligned}
$$

In particular, we find as well the simple expressions

$$
\begin{aligned}
\frac{\alpha \bar{\mu}_{2}}{\widetilde{\mu}_{2}}-\frac{\beta \bar{\mu}_{1}}{\widetilde{\mu}_{1}} & =\frac{\widetilde{\mu}_{3} \widetilde{M}_{4}+\mu_{3}^{*} \widetilde{M}_{3}}{\bar{\mu}_{3}}\left[\frac{\mu_{1}}{\widetilde{\mu}_{1}} \frac{\widetilde{M}_{1}}{\widetilde{M}}-\frac{\mu_{2}}{\widetilde{\mu}_{2}} \frac{\widetilde{M}_{2}}{\widetilde{M}}\right] \\
\gamma \bar{\mu}_{1} & =-\widetilde{\mu}_{1} \frac{\mu_{2}}{\bar{\mu}_{2}} \frac{\mu_{3}}{\bar{\mu}_{3}} \frac{\widetilde{M}}{\widetilde{M}}-\mu_{1}\left[\frac{\widetilde{\mu}_{2}}{\bar{\mu}_{2}} \frac{\mu_{3}}{\bar{\mu}_{3}} \frac{\widetilde{M}_{2}}{\widetilde{M}}+\frac{\mu_{2}}{\bar{\mu}_{2}} \frac{\widetilde{\mu}_{3} \widetilde{M}_{3}-\mu_{3} \widetilde{M}_{4}}{\bar{\mu}_{3} \widetilde{M}}\right],
\end{aligned}
$$

These expressions vanish trivially in the limit $\mu_{1}, \mu_{2} \rightarrow 0$ and then we obtain the limiting case discussed in Section 22. As already discussed in Section 3, $\beta \bar{\mu}_{1}$ is independent of $\mu_{2}$.

\section{A.1 Down Quarks and Charged Leptons}

Mass Eigenvalues and Eigenvectors. Now take the matrix $\bar{m}$ as a starting point and compute the eigenvalues, eigenvectors and mixing matrices. For making things simpler, consider for the moment all the parameters as complex, even if actually $\bar{\mu}_{3}$, $\bar{\mu}_{2}, \gamma \bar{\mu}_{1}$, or $\bar{\mu}_{3}, \bar{\mu}_{2}, \bar{\mu}_{1}$ can be chosen real absorbing the phases into $V_{3}$. To compute 
the eigenvalues, it is better to consider the hermitian matrices $\bar{m}^{\dagger} \bar{m}$ or $\bar{m} \bar{m}^{\dagger}$. The first option simply gives

$$
\bar{m}^{\dagger} \bar{m}=\left(\begin{array}{ccc}
\left|\bar{\mu}_{1}\right|^{2}|\gamma|^{2} & \left|\bar{\mu}_{1}\right|^{2} \gamma^{*} & \left|\bar{\mu}_{1}\right|^{2} \gamma^{*} \beta \\
\left|\bar{\mu}_{1}\right|^{2} \gamma & \left|\bar{\mu}_{2}\right|^{2}+\left|\bar{\mu}_{1}\right|^{2} & \left|\bar{\mu}_{2}\right|^{2} \alpha+\left|\bar{\mu}_{1}\right|^{2} \beta \\
\left|\bar{\mu}_{1}\right|^{2} \gamma \beta^{*}\left|\bar{\mu}_{2}\right|^{2} \alpha^{*}+\left|\bar{\mu}_{1}\right|^{2} \beta^{*} & \left|\bar{\mu}_{3}\right|^{2}+\left|\bar{\mu}_{2}\right|^{2}|\alpha|^{2}+\left|\bar{\mu}_{1}\right|^{2}|\beta|^{2}
\end{array}\right) .
$$

Then the determinant is simply

$$
\operatorname{det}\left(\bar{m}^{\dagger} \bar{m}\right)=|\operatorname{det}(\bar{m})|^{2}=|\gamma|^{2}\left|\bar{\mu}_{1}\right|^{2}\left|\bar{\mu}_{2}\right|^{2}\left|\bar{\mu}_{3}\right|^{2}
$$

and is only non-vanishing if $\gamma \bar{\mu}_{1} \neq 0$.

The eigenvalue equation is a cubic equation; to obtain the dominant terms, we expand around $\gamma=0$. In this case the equation reduces to a quadratic one with the solutions

$$
\begin{aligned}
\lambda_{2 / 3}= & \frac{1}{2}\left[\left|\bar{\mu}_{3}\right|^{2}+\left|\bar{\mu}_{2}\right|^{2}\left(1+|\alpha|^{2}\right)+\left|\bar{\mu}_{1}\right|^{2}\left(1+|\beta|^{2}\right)\right] \\
& \pm \frac{1}{2} \sqrt{\left[\left|\bar{\mu}_{3}\right|^{2}-\left|\bar{\mu}_{2}\right|^{2}\left(1-|\alpha|^{2}\right)-\left|\bar{\mu}_{1}\right|^{2}\left(1-|\beta|^{2}\right)\right]^{2}+\left.4|| \bar{\mu}_{2}\right|^{2} \alpha+\left.\left|\bar{\mu}_{1}\right|^{2} \beta\right|^{2}} .
\end{aligned}
$$

So in this limit, we have eigenvalues at lowest order

$$
\begin{array}{ll}
\lambda_{3}=\left|\bar{\mu}_{3}\right|^{2}+\left|\bar{\mu}_{2}\right|^{2}|\alpha|^{2}+\left|\bar{\mu}_{1}\right|^{2}|\beta|^{2}+\mathcal{O}\left(\frac{\lambda_{2}^{2}}{\lambda_{3}}\right), & \\
\lambda_{2}=\left|\bar{\mu}_{2}\right|^{2}+\left|\bar{\mu}_{1}\right|^{2}-\mathcal{O}\left(\frac{\lambda_{2}^{2}}{\lambda_{3}}\right), & \lambda_{1}=0 .
\end{array}
$$

We can also compute the first correction to the zero eigenvalue simply as

$$
\lambda_{1}=\frac{\operatorname{det}\left(\bar{m}^{\dagger} \bar{m}\right)}{\lambda_{2} \lambda_{3}}=\frac{|\gamma|^{2}\left|\bar{\mu}_{1}\right|^{2}\left|\bar{\mu}_{2}\right|^{2}\left|\bar{\mu}_{3}\right|^{2}}{\left|\bar{\mu}_{3}\right|^{2}\left(\left|\bar{\mu}_{2}\right|^{2}+\left|\bar{\mu}_{1}\right|^{2}\right)} \simeq \frac{|\gamma|^{2}\left|\bar{\mu}_{1}\right|^{2}\left|\bar{\mu}_{2}\right|^{2}}{\left|\bar{\mu}_{2}\right|^{2}+\left|\bar{\mu}_{1}\right|^{2}} \stackrel{\left|\bar{\mu}_{1}\right| \ll\left|\bar{\mu}_{2}\right|}{\longrightarrow}|\gamma|^{2}\left|\bar{\mu}_{1}\right|^{2} \text {. }
$$

This means that for vanishing $\mu_{1}$ we have

$$
m_{d} \simeq|\gamma|\left|\bar{\mu}_{1}\right| \simeq \frac{\left|\mu_{2}\right|}{\left|\bar{\mu}_{2}\right|}\left|\widetilde{\mu}_{1}\right|
$$

Using the eigenvalues, we can also solve for the mixing matrices at lowest order,

$$
V_{3}^{\prime}=\left(\begin{array}{ccc}
1 & 0 & 0 \\
0 & 1 & \frac{\left|\bar{\mu}_{2}\right|^{2} \alpha+\left|\bar{\mu}_{1}\right|^{2} \beta}{\left|\bar{\mu}_{3}\right|} \\
0-\frac{\left|\bar{\mu}_{2}\right|^{2} \alpha^{*}+\left|\bar{\mu}_{1}\right|^{2} \beta^{*}}{\left|\bar{\mu}_{3}\right|} & 1
\end{array}\right),
$$

where we must recall that we had already acted on the mass matrix with a large angle rotation $\widehat{V}_{3}$, so the $V_{3}^{\prime}$ above is just a small correction to it. 
For the left-handed quark fields, we have instead at leading order

$$
U_{3}=\left(\begin{array}{ccc}
1 & \bar{\mu}_{1} & \frac{\bar{\mu}_{1} \beta}{\bar{\mu}_{3}} \\
-\frac{\bar{\mu}_{1}^{*}}{\bar{\mu}_{2}^{*}} & 1 & \frac{\bar{\mu}_{2} \alpha}{\bar{\mu}_{3}} \\
\frac{\bar{\mu}_{1}^{*}}{\bar{\mu}_{3}^{*}}\left(\alpha^{*}-\beta^{*}\right) & -\frac{\bar{\mu}_{2}^{*} \alpha^{*}}{\bar{\mu}_{3}^{*}} & 1
\end{array}\right) .
$$

Since the up quark mass matrix is already diagonal, this last mixing matrix corresponds to the CKM matrix. From $U_{3}^{\dagger} \bar{m} V_{3}^{\prime}=m^{\text {diag }}$, we get $V_{\mathrm{CKM}}=U_{3}$, so for $\alpha=\beta$ we have the prediction $V_{t d}=\left(\alpha^{*}-\beta^{*}\right) \bar{\mu}_{1}^{*} / \bar{\mu}_{3}^{*}=0$ at leading order, and the $\mathrm{CP}$ violation vanishes! On the other hand, $V_{u b}$ has the right order of magnitude as we thought.

Quark Masses and Mixing Angles. We can reproduce the observed quark mass eigenvalues and mixing, that satisfy the relations

$$
\begin{aligned}
& m_{u}: m_{c}: m_{t} \simeq \lambda^{7}: \lambda^{3}: 1 \\
& m_{d}: m_{s}: m_{b} \simeq \lambda^{4}: \lambda^{2}: 1
\end{aligned}
$$

where $\lambda \simeq V_{u s} \sim 0.22$ is the Cabibbo angle. In fact, if we assume

$$
\begin{aligned}
& \mu_{1}: \mu_{2}: \mu_{3} \simeq \lambda^{7}: \lambda^{3}: 1, \\
& \widetilde{\mu}_{1}: \widetilde{\mu}_{2}: \widetilde{\mu}_{3} \simeq \lambda^{3}: \lambda^{2}: 1,
\end{aligned}
$$

it gives correctly

$$
\begin{aligned}
& \left|V_{u s}\right| \sim \frac{\left|\bar{\mu}_{1}\right|}{\left|\bar{\mu}_{2}\right|} \sim \frac{\left|\widetilde{\mu}_{1}\right|}{\left|\widetilde{\mu}_{2}\right|} \sim \lambda, \\
& \left|V_{u b}\right| \sim \frac{\left|\bar{\mu}_{1}\right|}{\left|\bar{\mu}_{3}\right|} \sim \frac{\left|\widetilde{\mu}_{1}\right|}{\left|\widetilde{\mu}_{3}\right|} \sim \lambda^{3}, \quad\left|V_{c b}\right| \sim \frac{\left|\bar{\mu}_{2}\right|}{\left|\bar{\mu}_{3}\right|} \sim \frac{\left|\widetilde{\mu}_{2}\right|}{\left|\widetilde{\mu}_{3}\right|} \sim \lambda^{2} ;
\end{aligned}
$$

moreover,

$$
m_{d} \simeq \frac{|\gamma|}{\sqrt{1+|\alpha|^{2}}}\left|\bar{\mu}_{1}\right| \simeq \frac{\left|\mu_{2}\right|}{\left|\bar{\mu}_{2}\right|} \frac{\left|\widetilde{\mu}_{1}\right|}{\bar{\mu}_{3}} m_{b} \simeq \lambda \lambda^{3} m_{b} \simeq \lambda^{4} m_{b}
$$

Again $V_{t d}$ is suppressed by the difference of $\alpha^{*}-\beta^{*} \simeq \mu_{2} / \bar{\mu}_{2}, \mu_{1} / \bar{\mu}_{1}$, as is the Jarlskog invariant, $J_{q}$.

Low-energy CP violation As discussed in the following Appendix, we can express the low-energy $\mathrm{CP}$ violation in the quark section via an effective Jarlskog invariant. We calculate this invariant, using Eqs. (A.4). The dominant terms are 
displayed in Eq. (3.9); the complete expression reads

$$
\begin{aligned}
& J_{q}=\frac{\widetilde{\mu}_{1}^{2} \widetilde{\mu}_{2}^{2} \widetilde{\mu}_{3}^{2}}{\Delta \mathscr{M}_{d}^{2}}\left\{\frac{\widetilde{M}_{2} \widetilde{M}_{3}}{\widetilde{M}^{2}}\left[\left(1-\frac{\widetilde{M}_{4}^{2}}{\widetilde{M}^{2}}\right) \operatorname{Im} \frac{\mu_{3} \mu_{2}^{*}}{\widetilde{\mu}_{3} \widetilde{\mu}_{2}^{2}}+\frac{\widetilde{M}_{3} \widetilde{M}_{4}}{\widetilde{M}^{2}} \frac{\left|\mu_{3}\right|^{2}}{\widetilde{\mu}_{3}^{2}} \operatorname{Im} \frac{\mu_{2}}{\widetilde{\mu}_{2}}\right]\right. \\
& -\frac{\widetilde{M}_{2}^{2} \widetilde{M}_{3} \widetilde{M}_{4}}{\widetilde{M}^{4}} \frac{\left|\mu_{2}\right|^{2}}{\widetilde{\mu}_{2}^{2}} \operatorname{Im} \frac{\mu_{3}}{\widetilde{\mu}_{3}} \\
& -\frac{\widetilde{M}_{1} \widetilde{M}_{3}}{\widetilde{M}^{2}}\left[\left(1-\frac{\widetilde{M}_{4}^{2}}{\widetilde{M}^{2}}\right) \operatorname{Im} \frac{\mu_{3} \mu_{1}^{*}}{\widetilde{\mu}_{3} \widetilde{\mu}_{1}}+\frac{\widetilde{M}_{3} \widetilde{M}_{4}}{\widetilde{M}^{2}} \frac{\left|\widetilde{\mu}_{3}\right|^{2}}{\widetilde{\mu}_{3}^{2}} \operatorname{Im} \frac{\mu_{1}}{\widetilde{\mu}_{1}}\right] \\
& +\frac{\widetilde{M}_{1} \widetilde{M}_{2}}{\widetilde{M}^{2}}\left[\left(1-\frac{\widetilde{M}_{4}^{2}}{\widetilde{M}^{2}}\right)+\frac{\widetilde{M}_{3}^{2}}{\widetilde{M}^{2}} \frac{\left|\mu_{3}\right|^{2}}{\widetilde{\mu}_{3}^{2}}\right] \operatorname{Im} \frac{\mu_{2} \mu_{1}^{*}}{\widetilde{\mu}_{2} \widetilde{\mu}_{1}} \\
& +\frac{\widetilde{M}_{1} \widetilde{M}_{2}^{2}}{\widetilde{M}^{3}} \frac{\left|\mu_{2}\right|^{2}}{\widetilde{\mu}_{2}^{2}}\left[\frac{\widetilde{M}_{4}}{\widetilde{M}} \operatorname{Im} \frac{\mu_{1}}{\widetilde{\mu}_{1}}-\frac{\widetilde{M}_{3}}{\widetilde{M}} \operatorname{Im} \frac{\mu_{3} \mu_{1}^{*}}{\widetilde{\mu}_{3} \widetilde{\mu}_{1}}\right] \\
& \left.+\frac{\widetilde{M}_{1}^{2}}{\widetilde{M}^{2}} \frac{\left|\mu_{1}\right|^{2}}{\widetilde{\mu}_{1}^{2}}\left[\frac{\widetilde{M}_{3} \widetilde{M}_{4}}{\widetilde{M}^{2}} \operatorname{Im} \frac{\mu_{3}}{\widetilde{\mu}_{3}}-\frac{\widetilde{M}_{2} \widetilde{M}_{4}}{\widetilde{M}^{2}} \operatorname{Im} \frac{\mu_{2}}{\widetilde{\mu}_{2}}+\frac{\widetilde{M}_{2} \widetilde{M}_{3}}{\widetilde{M}^{2}} \operatorname{Im} \frac{\mu_{3} \mu_{2}^{*}}{\widetilde{\mu}_{3} \widetilde{\mu}_{2}}\right]\right\} .
\end{aligned}
$$

Charged Leptons. The charged leptons show a different hierarchy than the down quarks, we have in fact

$$
\begin{aligned}
& m_{e}: m_{\mu}: m_{\tau} \simeq \lambda^{5-6}: \lambda^{2}: 1 \\
& m_{d}: m_{s}: m_{b} \simeq \lambda^{4}: \lambda^{2}: 1 .
\end{aligned}
$$

The discrepancy can be solved with a smaller value for $\left(\mu_{2} \tilde{\mu}_{1}\right)_{e}$, compared to $\left(\mu_{2} \tilde{\mu}_{1}\right)_{d}$. As an example, we choose $\mu_{2}^{e} \simeq \lambda^{4}$ and $\widetilde{\mu}_{1}^{e} \simeq \lambda^{3-4}$ such that

$$
m_{e} \simeq \frac{\left|\gamma^{e}\right|}{\sqrt{1+|\alpha|^{2}}}\left|\bar{\mu}_{1}\right| \simeq \frac{\left|\mu_{2}^{e}\right|}{\left|\bar{\mu}_{2}\right|} \mid \frac{\widetilde{\mu}_{1}^{e} \mid}{\bar{\mu}_{3}} m_{\tau} \simeq \lambda^{2} \lambda^{3-4} m_{\tau} \simeq \lambda^{5-6} m_{\tau} .
$$

Regarding the rotations, the large $V_{4}$ rotation acts now on the left-handed fields, but it has to act on both the charged leptons and the neutrinos, so it has not a large effect in the charged current. There is, however, an effect coming from the mismatch between the two $V_{3}$ 's in the charged leptons and neutrino cases.

\section{A.2 Neutrinos}

The charged lepton mass matrix is eventually diagonalised via $V_{3}=\widehat{V}_{3} V_{3}^{\prime}$ and $U_{3}$ as the down quark matrix. For the light neutrino Majorana mass matrix, given by

$$
m_{\mathrm{eff}}^{\nu}=-\left(m^{D}\right)^{\top}\left(m^{N}\right)^{-1} m^{D},
$$

we can neglect the rotation $U_{3}$ of the right-handed fields as this transformation cancels out. $U_{4}$ does in principle rotate the RH states, but its effect is suppressed as long as $M_{i}<\tilde{M}$. Regarding $V_{3}$, we do not expect it to be the same for both charged 
and neutral leptons, so the mismatch between the two provides flavour mixing in the neutrino sector.

The neutrino Dirac mass matrix can be written after the large rotation $\widehat{V}_{3}$ that bring the charged lepton mass matrix into triangular form as

$$
\bar{m}^{D}=\widehat{m}^{D} \widehat{V}_{3}=\left(\begin{array}{ccc}
A \bar{\rho}_{1} & D \bar{\rho}_{1} & \bar{\rho}_{1} \\
B \bar{\rho}_{2} & E \bar{\rho}_{2} & \bar{\rho}_{2} \\
C \bar{\rho}_{3} & F \bar{\rho}_{3} & \bar{\rho}_{3}
\end{array}\right),
$$

where

$$
\begin{aligned}
& \bar{\rho}_{1}=\frac{1}{\bar{\mu}_{3}} \frac{1}{\widetilde{M}^{2}}\left\{\widetilde{\rho}_{1}\left[\widetilde{\mu}_{3} \widetilde{M}_{123}^{2}-\mu_{3}^{*} \widetilde{M}_{3} \widetilde{M}_{4}\right]-\rho_{1} \widetilde{M}_{1}\left[\widetilde{\mu}_{3} \widetilde{M}_{4}+\mu_{3}^{*} \widetilde{M}_{3}\right]\right\} \\
& \bar{\rho}_{2}=\frac{1}{\bar{\mu}_{3}} \frac{1}{\widetilde{M}^{2}}\left\{\widetilde{\rho}_{2}\left[\widetilde{\mu}_{3} \widetilde{M}_{123}^{2}-\mu_{3}^{*} \widetilde{M}_{3} \widetilde{M}_{4}\right]-\rho_{2} \widetilde{M}_{2}\left[\widetilde{\mu}_{3} \widetilde{M}_{4}+\mu_{3}^{*} \widetilde{M}_{3}\right]\right\}, \\
& \bar{\rho}_{3}=\frac{1}{\bar{\mu}_{3}} \frac{1}{\widetilde{M}^{2}}\left\{\widetilde{\rho}_{3}\left[\widetilde{\mu}_{3} \widetilde{M}_{123}^{2}-\mu_{3}^{*} \widetilde{M}_{3} \widetilde{M}_{4}\right]-\rho_{3}\left[\widetilde{\mu}_{3} \widetilde{M}_{3} \widetilde{M}_{4}-\mu_{3}^{*} \widetilde{M}_{124}^{2}\right]\right\},
\end{aligned}
$$

and, using the notation $\widetilde{M}_{\alpha \beta}=\sqrt{\widetilde{M}_{\alpha}^{2}+\widetilde{M}_{\beta}^{2}}$,

$$
\begin{aligned}
& A=-\frac{1}{\bar{\rho}_{1}} \frac{1}{\bar{\mu}_{2}} \frac{1}{\bar{\mu}_{3}} \frac{1}{\widetilde{M}}\left\{\widetilde{\rho}_{1} \mu_{2} \mu_{3} \widetilde{M}_{1}-\rho_{1}\left[\widetilde{\mu}_{2} \mu_{3} \widetilde{M}_{2}+\mu_{2}\left(\widetilde{\mu}_{3} \widetilde{M}_{3}-\mu_{3} \widetilde{M}_{4}\right)\right]\right\} \\
& B=\frac{\rho_{2} \widetilde{\mu}_{2}-\widetilde{\rho}_{2} \mu_{2}}{\bar{\rho}_{2} \bar{\mu}_{2}} \frac{\mu_{3}}{\bar{\mu}_{3}} \frac{\widetilde{M}_{1}}{\widetilde{M}} \\
& C=\frac{\widetilde{\mu}_{3} \rho_{3}-\mu_{3} \widetilde{\rho}_{3}}{\bar{\rho}_{3} \bar{\mu}_{3}} \frac{\mu_{2}}{\bar{\mu}_{2}} \frac{\widetilde{M}_{1}}{\widetilde{M}} \\
& D=\frac{1}{\bar{\rho}_{1}} \frac{1}{\bar{\mu}_{2}} \frac{1}{\bar{\mu}_{3}^{2}} \frac{1}{\widetilde{M}^{2}}\left\{\widetilde{\rho}_{1}\left[\widetilde{\mu}_{2}\left|\mu_{3}\right|^{2} \widetilde{M}_{12}^{2}+\mu_{2}^{*} \mu_{3} \widetilde{M}_{2}\left(\widetilde{\mu}_{3} \widetilde{M}_{3}-\mu_{3}^{*} \widetilde{M}_{4}\right)\right]\right. \\
& \left.+\rho_{1} \widetilde{M}_{1}\left[\widetilde{\mu}_{2} \mu_{3}^{*}\left(\mu_{3} \widetilde{M}_{4}-\widetilde{\mu}_{3} \widetilde{M}_{3}\right)+\mu_{2}^{*} \widetilde{M}_{2}\left(\widetilde{\mu}_{3}^{2}+\left|\mu_{3}\right|^{2}\right)\right]\right\}, \\
& E=\frac{1}{\bar{\rho}_{2}} \frac{1}{\bar{\mu}_{2}} \frac{1}{\bar{\mu}_{3}^{2}} \frac{1}{\widetilde{M}^{2}}\left\{\widetilde{\rho}_{2}\left[\widetilde{\mu}_{2}\left|\mu_{3}\right|^{2} \widetilde{M}_{12}^{2}+\mu_{2}^{*} \mu_{3} \widetilde{M}_{2}\left(\widetilde{\mu}_{3} \widetilde{M}_{3}-\mu_{3}^{*} \widetilde{M}_{4}\right)\right]\right. \\
& +\rho_{2}\left[\widetilde{\mu}_{2} \mu_{3}^{*} \widetilde{M}_{2}\left(\widetilde{\mu}_{3} \widetilde{M}_{3}-\mu_{3} \widetilde{M}_{4}\right)\right. \\
& \left.\left.+\mu_{2}^{*}\left(\widetilde{\mu}_{3}^{2} \widetilde{M}_{13}^{2}-2\left|\mu_{3}\right| \widetilde{\mu}_{3} \widetilde{M}_{3} \widetilde{M}_{4} \cos \theta_{3}+\left|\mu_{3}\right|^{2} \widetilde{M}_{14}^{2}\right)\right]\right\}, \\
& F=\frac{1}{\bar{\rho}_{3}} \frac{1}{\bar{\mu}_{2}} \frac{1}{\bar{\mu}_{3}^{2}} \frac{1}{\widetilde{M}^{2}}\left(\widetilde{\rho}_{3} \mu_{3}-\rho_{3} \widetilde{\mu}_{3}\right)\left[\widetilde{\mu}_{2} \mu_{3}^{*} \widetilde{M}_{12}^{2}+\mu_{2}^{*} \widetilde{M}_{2}\left(\widetilde{\mu}_{3} \widetilde{M}_{3}-\mu_{3}^{*} \widetilde{M}_{4}\right)\right] .
\end{aligned}
$$

Note that we are here projecting the neutrino flavour states into the basis defined by the charged leptons as in Eq. (A.3). So we can immediately see that if the neutrino flavour vectors are aligned with the charged leptons $B, C, F$ should vanish and the neutrino mass matrix would become triangular as well. This corresponds to having 
exactly the same hierarchy in the rows of the charged and neutral lepton Dirac mass matrices, i.e. $\frac{\mu_{i}}{\widetilde{\mu}_{i}}=\frac{\rho_{i}}{\widetilde{\rho}_{i}}$. We do not expect such alignment since the parameters $\widetilde{\rho}_{i}, \widetilde{\mu}_{i}$ are generated by different operators and not related by any GUT relation, as can be seen from Eq. (2.3c). We will consider in the following the case where the neutrino hierarchies are similar to those of the down quark matrix, while the charged leptons differ due to the lighter electron mass. Of course even more involved scenarios are possible. In the following we neglect as well corrections coming from the final diagonalisation, since the entries of $V_{3}^{\prime}$ are suppressed by $\left(\mu_{2} / \bar{\mu}_{2}\right)^{2} \lesssim 0.01$.

Mass eigenvalues and eigenvectors. We need to compute the eigenvalues of the neutrino mass matrix and the first step is again to compute the determinant of the matrix $m_{\text {eff }}^{\nu}$. Note that this is a symmetric matrix, but not real. Therefore the eigenvalues are in general complex and the matrix is diagonalised using a unitary matrix $V_{\nu}$ as

$$
V_{\nu}^{\top} m_{\mathrm{eff}}^{\nu} V_{\nu}=\operatorname{diag}\left(m_{1}, m_{2}, m_{3}\right) .
$$

Consider for the moment just the absolute value of the eigenvalues and then see that we have the relation ${ }^{3}$

$$
\operatorname{det}\left(m_{\mathrm{eff}}^{\nu}\right)=-\frac{\left(\operatorname{det}\left(m^{D}\right)\right)^{2}}{\operatorname{det}\left(m^{N}\right)}
$$

The last determinant is simply the product of the heavy neutrino masses, while the first one is given by

$$
\operatorname{det}\left(m^{D}\right)=\bar{\rho}_{1} \bar{\rho}_{2} \bar{\rho}_{3}[(F-E)(A-B)+(D-E)(B-C)] .
$$

In order to have three non-vanishing eigenvalues, we need all $\bar{\rho}_{i} \neq 0$ and at least one of $A, B$, and $C$ different from zero. Also the three vectors corresponding to the rows of the Dirac matrix must not be aligned with each other. So we obtain

$$
\begin{aligned}
& m_{1} m_{2} m_{3}=-\varrho_{1} \varrho_{2} \varrho_{3} {[(F-E)(A-B)+(D-E)(B-C)]^{2} } \\
&=-\varrho_{1} \varrho_{2} \varrho_{3} \frac{\widetilde{\rho}_{1}}{\bar{\rho}_{1}} \frac{\rho_{2}}{\bar{\rho}_{2}} \frac{\rho_{3}}{\bar{\rho}_{3}} \frac{1}{\bar{\mu}_{2}^{2}} \frac{1}{\bar{\mu}_{3}^{2}} \frac{1}{\widetilde{M}^{2}} \frac{\widetilde{M}_{1}}{\widetilde{M}} \\
& \quad \times\left\{\widetilde{\mu}_{2}^{2}\left|\mu_{3}\right|^{2} \widetilde{M}_{12}^{2}+2\left|\mu_{2}\right| \widetilde{\mu}_{2}\left|\mu_{3}\right| \widetilde{M}_{2}\left[\widetilde{\mu}_{3} \widetilde{M}_{3} \cos \left(\theta_{2}-\theta_{3}\right)-\mu_{3} \widetilde{M}_{4} \cos \theta_{2}\right]\right. \\
&\left.\quad+\left|\mu_{2}\right|^{2}\left[\widetilde{\mu}_{3}^{2} \widetilde{M}_{13}^{2}-2\left|\mu_{3}\right| \widetilde{\mu}_{3} \widetilde{M}_{3} \widetilde{M}_{4} \cos \theta_{3}+\left|\mu_{3}\right|^{2} \widetilde{M}_{14}^{2}\right]\right\}
\end{aligned}
$$

for $\rho_{1}=0$, where $\varrho_{i}=e^{-2 i \phi_{i}} \bar{\rho}_{i}^{2} / M_{i}$.

\footnotetext{
${ }^{3}$ Note that for a $n \times n$ mass matrix, the minus sign on the r.h.s. gives a $(-1)^{n}$ contribution.
} 
Singling out the heaviest mass eigenstate. In the case when $\varrho_{3} \gg \varrho_{2,1}$, it is easy to single out the heaviest eigenstate:

$$
\left(v_{\nu, 3}^{0}\right)^{\top}=\frac{1}{\sqrt{1+|F|^{2}+|C|^{2}}}\left(C^{*}, F^{*}, 1\right)
$$

and the mass eigenvalue to lowest order is given by

$$
m_{3}^{0}=-\varrho_{3}\left(1+|F|^{2}+|C|^{2}\right) .
$$

Then up to a rotation in the 12 submatrix, at lowest order the mixing matrix can be written as

$$
V_{\nu}^{0}=\left(\begin{array}{ccc}
\frac{\sqrt{1+|F|^{2}}}{\sqrt{1+|F|^{2}+|C|^{2}}} & 0 & \frac{C^{*}}{\sqrt{1+|F|^{2}+|C|^{2}}} \\
\frac{-C F^{*}}{\sqrt{1+|F|^{2}+|C|^{2}} \sqrt{1+|F|^{2}}} & \frac{1}{\sqrt{1+|F|^{2}}} \frac{F^{*}}{\sqrt{1+|F|^{2}+|C|^{2}}} \\
\frac{-C}{\sqrt{1+|F|^{2}+|C|^{2}} \sqrt{1+|F|^{2}}} & \frac{-F}{\sqrt{1+|F|^{2}}} & \frac{1}{\sqrt{1+|F|^{2}+|C|^{2}}}
\end{array}\right)
$$

this is the basis which gives decoupling of the first eigenstate in the limit of vanishing $C$. From this matrix, we can directly read off the dominant part of the mixing angles with the heavy eigenstate, $\theta_{23}$ and $\theta_{13}$. The charged lepton mass matrix is nearly diagonal, so we can actually relate with good accuracy the first row to the electron neutrino flavour. The left-handed charged lepton flavour eigenstates are given as a function of the mass eigenstates by

$$
\ell_{f}=\left(\widehat{V}_{3} V_{3}^{\prime}\right)^{\dagger} \ell_{i}
$$

and therefore the neutrino flavour eigenstates correspond to

$$
\nu_{f}=\left(\widehat{V}_{3} V_{3}^{\prime}\right)^{\dagger} \widehat{V}_{3} V_{\nu} \nu_{i}=\left(V_{3}^{\prime}\right)^{\dagger} V_{\nu} \nu_{i}
$$

where $\widehat{V}_{3}$ cancels out as it acts equally on the whole lepton doublet; moreover, as we have seen, $V_{3}^{\prime}$ is limited to the 23 corner and does not modify the electron entry. We use here the convention of [32], and define the PMNS matrix as

$$
\begin{aligned}
V_{\nu} & =\left(\begin{array}{ccc}
c_{13} c_{12} & c_{13} s_{12} & s_{13} \\
-s_{12} c_{23}-c_{12} s_{23} s_{13} e^{i \delta} & c_{12} c_{23}-s_{12} s_{23} s_{13} e^{i \delta} & c_{13} s_{23} e^{i \delta} \\
s_{12} s_{23}-c_{12} c_{23} s_{13} e^{i \delta} & -c_{12} s_{23}-s_{12} c_{23} s_{13} e^{i \delta} & c_{13} c_{23} e^{i \delta}
\end{array}\right)\left(\begin{array}{ccc}
1 & 0 & 0 \\
0 & e^{i \xi_{2} / 2} & 0 \\
0 & 0 & e^{i \xi_{3} / 2}
\end{array}\right) \\
& =\left(\begin{array}{ccc}
1 & 0 & 0 \\
0 & c_{23} & s_{23} \\
0 & -s_{23} & c_{23}
\end{array}\right)\left(\begin{array}{ccc}
c_{13} & 0 & s_{13} e^{-i \delta} \\
0 & 1 & 0 \\
-s_{13} e^{i \delta} & 0 & c_{13}
\end{array}\right)\left(\begin{array}{ccc}
c_{12} & s_{12} & 0 \\
-s_{12} & c_{12} & 0 \\
0 & 0 & 1
\end{array}\right)\left(\begin{array}{ccc}
1 & 0 & 0 \\
0 & e^{i \xi_{2} / 2} & 0 \\
0 & 0 & e^{i\left(\delta+\xi_{3} / 2\right)}
\end{array}\right),
\end{aligned}
$$

where $c_{i j}=\cos \theta_{i j}, s_{i j}=\sin \theta_{i j}, \delta$ is the Dirac phase and $\xi_{1,2}$ are the Majorana phases. 
So we have at lowest order for $\theta_{13}$ that

$$
\left(V_{\nu}^{0}\right)_{13}=\sin \theta_{13} \simeq \frac{|C|}{\sqrt{1+|F|^{2}+|C|^{2}}} .
$$

This gives us directly a constraint on the parameter $C$ from the upper bound on $\left|\sin \theta_{13}\right| \leq 0.1$ :

$$
|C| \simeq \sqrt{1+|F|^{2}+|C|^{2}}\left|\sin \theta_{13}\right| \lesssim 0.1 \sqrt{1+|F|^{2}} .
$$

Then since the mixing with the first flavour is small, the atmospheric mixing matrix is given simply by requiring the 23 corner of the matrix in Eq. (A.31) to give

$$
V_{\mathrm{atm}, 23}=\left(\begin{array}{cc}
\cos \theta_{23} & \sin \theta_{23} e^{-i \xi_{23}} \\
-\sin \theta_{23} e^{i \xi_{23}} & \cos \theta_{23}
\end{array}\right) .
$$

So considering the 23 sector, we get, again at lowest order,

$$
\begin{aligned}
\xi_{23} & =\arg (F), \\
\tan \theta_{23} & =|F| .
\end{aligned}
$$

To have large mixing angle $\tan 2 \theta_{23} \geq 3[29,31]$, we must restrict $|F|$ between

$$
0.7 \leq|F| \leq 1.4 \text {. }
$$

Such a value is natural in the case where $\rho_{3}, \widetilde{\rho}_{3}$ and $\mu_{3}, \widetilde{\mu}_{3}$ are of the same order but not exactly equal, while $\mu_{2}$ is small. Note that even a phase difference can be important. Assuming simply $\frac{\rho_{3}}{\widetilde{\rho}_{3}}=e^{i \omega_{3}} \frac{\mu_{3}}{\widetilde{\mu}_{3}}$ and degenerate $\widetilde{M}_{i}$ gives

$$
|F|=\frac{2 \sqrt{2\left(1-\cos \omega_{3}\right)}}{3-\cos \omega_{3}}
$$

so we obtain $|F|=1$ for the maximal phase difference $\omega_{3}=\pi$, while $|F| \geq 0.7$ arises in the wide interval $0.26 \pi \leq \omega_{3} \leq 1.73 \pi$. Hence, a nearly maximal atmospheric angle is natural even for the most simple choice of parameters. Of course, more solutions are possible for the general case.

Thus in order to reproduce the observed pattern of mixing parameters, $C$ has to be small, while $|F|$ is nearly unity. We can use the maximal value for $|F|$ and the experimental bound on $\theta_{13}$ to derive an upper limit on $|C|$,

$$
|C| \leq 0.17
$$

in agreement e.g. with the ratio $\frac{\mu_{2}}{\widetilde{\mu}_{2}}$ necessary to have a small electron mass. Note, however, that we can obtain significant corrections from $\varrho_{2,1} \neq 0$. 
Light eigenstates and solar mixing angle. The other two eigenvalues and the correction to the heavy mass can be obtained from the trace and determinant of the matrix $\left(m_{\text {eff }}^{\nu}\right)^{\dagger} m_{\text {eff }}^{\nu}$, which can be computed in any basis. Expanding both the mass matrix and the eigenvalues to first order,

$$
\begin{aligned}
m_{\mathrm{eff}}^{\nu} & =m_{\varrho_{3}}+m_{\varrho_{1,2}}, \\
m_{3} & =m_{3}^{0}+\delta m_{3} \quad \text { while } \quad m_{1,2}=\delta m_{1,2},
\end{aligned}
$$

we have then

$$
\begin{aligned}
\delta m_{3}=\frac{\operatorname{tr}\left[m_{\varrho_{3}}^{\dagger} m_{\varrho_{1,2}}\right]}{\left(m_{3}^{0}\right)^{*}}, \quad\left|m_{1}\right|^{2}+\left|m_{2}\right|^{2}+\left|\delta m_{3}\right|^{2}=\operatorname{tr}\left[m_{\varrho_{1,2}}^{\dagger} m_{\varrho_{1,2}}\right], \\
\left|m_{2}\right|^{2}\left|m_{1}\right|^{2}=\frac{\left|\operatorname{det}\left(m_{\mathrm{eff}}^{\nu}\right)\right|^{2}}{\left|m_{3}\right|^{2}} .
\end{aligned}
$$

Choosing the basis appropriately, the relations can be simplified to give

$$
\begin{aligned}
\delta m_{3} & =\left(\left(V_{\nu}^{0}\right)^{\top} m_{\varrho_{1,2}} V_{\nu}^{0}\right)_{33} \\
\left|m_{1}\right|^{2}+\left|m_{2}\right|^{2} & =\operatorname{tr}\left[m_{\varrho_{1,2}}^{\dagger} m_{\varrho_{1,2}}\right]-\left|\left(\left(V_{\nu}^{0}\right)^{\top} m_{\varrho_{1,2}} V_{\nu}^{0}\right)_{33}\right|^{2} \\
\left|m_{2}\right|^{2}\left|m_{1}\right|^{2} & \sim\left|\varrho_{1} \varrho_{2}\right|^{2} \frac{|(F-E)(A-B)+(D-E)(B-C)|^{2}}{\left(1+|F|^{2}+|C|^{2}\right)^{2}}
\end{aligned}
$$

We will give the result of these expressions for vanishing $C$ and $\varrho_{1}=q \varrho_{2}$ :

$$
\begin{gathered}
\delta m_{3}=\varrho_{2} \frac{(1-F E)^{2}+q(1-F D)^{2}}{1+|F|^{2}} \\
\operatorname{tr}\left[m_{\varrho_{1,2}}^{\dagger} m_{\varrho_{1,2}}\right]=\left|\varrho_{2}\right|^{2}\left[|1+q|^{2}+\left|E^{2}+q D^{2}\right|^{2}+\left|B^{2}+q A^{2}\right|^{2}\right. \\
\left.+2|B E+q A D|^{2}+2|B+q A|^{2}+2|E+q D|^{2}\right], \\
\left|m_{2}\right|^{2}\left|m_{1}\right|^{2} \sim\left|\varrho_{2}\right|^{4}|q|^{2} \frac{|A(F-E)+B(D-F)|^{4}}{\left(1+|F|^{2}\right)^{2}}
\end{gathered}
$$

Then the mass splitting which should generate the solar oscillations is given by

$$
\begin{gathered}
\delta m_{\mathrm{sol}}^{2}=\sqrt{\left(\left|m_{1}\right|^{2}+\left|m_{2}\right|^{2}\right)^{2}-4\left|m_{2}\right|^{2}\left|m_{1}\right|^{2}} \\
=\frac{\left|\varrho_{2}\right|^{2}}{\left(1+|F|^{2}\right)^{2}}\left\{\left[( 1 + | F | ^ { 2 } ) ^ { 2 } \left(|1+q|^{2}+\left|E^{2}+q D^{2}\right|^{2}+\left|B^{2}+q A^{2}\right|^{2}\right.\right.\right. \\
\left.+2|B E+q A D|^{2}+2|B+q A|^{2}+2|E+q D|^{2}\right) \\
\left.-\left|(1-F E)^{2}+q(1-F D)^{2}\right|^{2}\right]^{2} \\
\left.-4|q|^{2}\left(1+|F|^{2}\right)^{2}|A(F-E)+B(D-F)|^{4}\right\}^{1 / 2} .
\end{gathered}
$$


So the solar neutrino mass splitting can be matched even in the case $q=0$ or $A(F-E)+B(D-F)=0$, i.e., when the lightest neutrino is massless. However, we do not expect the first limit to be realised, if we assume the same hierarchies between $\bar{\rho}_{i}$ as in the $\bar{\mu}_{i}$ in the down quark sector, while for $M_{i}$ as the up quark sector. In that case we have in fact $\left|\varrho_{2}\right| \sim\left|\varrho_{1}\right|$ and the two lighter eigenvalues are similar in scale, $m_{1} \simeq m_{2} \simeq \sqrt{\delta m_{\text {sol }}^{2}}$. On the other hand, the determinant could be suppressed by alignment, i.e., for $|A(F-E)+B(D-F)| \ll 1$, and could give us a hierarchy also between the two light eigenvalues.

We can then compute the solar mixing angle and the first order corrections to the $V_{e 3}$ mixing parameter. After rotating with the $V_{\nu}^{0}$ matrix, we can estimate the solar angle by using only the 12 part of the mass matrix; for $C \simeq 0$ the matrix is given by

$$
m_{\varrho_{1,2}}(12)=\left(\begin{array}{cc}
B^{2} \varrho_{2}+A^{2} \varrho_{1} & B \varrho_{2} \frac{E-F}{\sqrt{1+|F|^{2}}}+A \varrho_{1} \frac{D-F}{\sqrt{1+|F|^{2}}} \\
B \varrho_{2} \frac{E-F}{\sqrt{1+|F|^{2}}}+A \varrho_{1} \frac{D-F}{\sqrt{1+|F|^{2}}} & \varrho_{2} \frac{(E-F)^{2}}{1+|F|^{2}}+\varrho_{1} \frac{(D-F)^{2}}{1+|F|^{2}}
\end{array}\right)
$$

Taking the solar mixing matrix as in Eq. A.37) with $\theta_{23}, \xi_{23} \rightarrow \theta_{12}, \xi_{12}$ we obtain

$$
\begin{aligned}
e^{-i \xi_{12}} & =\frac{\left(m_{\varrho_{1,2}}\right)_{12}\left(m_{\varrho_{1,2}}\right)_{11}^{*}+\left(m_{\varrho_{1,2}}\right)_{22}\left(m_{\varrho_{1,2}}\right)_{12}^{*}}{\left|\left(m_{\varrho_{1,2}}\right)_{12}\left(m_{\varrho_{1,2}}\right)_{11}^{*}+\left(m_{\varrho_{1,2}}\right)_{22}\left(m_{\varrho_{1,2}}\right)_{12}^{*}\right|} \\
\tan 2 \theta_{12} & =\frac{2\left|\left(m_{\varrho_{1,2}}\right)_{12}\left(m_{\varrho_{1,2}}\right)_{11}^{*}+\left(m_{\varrho_{1,2}}\right)_{22}\left(m_{\varrho_{1,2}}\right)_{12}^{*}\right|}{\left|\left(m_{\varrho_{1,2}}\right)_{22}\right|^{2}-\left|\left(m_{\varrho_{1,2}}\right)_{11}\right|^{2}}=\frac{2 \sqrt{1+|F|^{2}}|\mathcal{N}|}{\mathcal{D}},
\end{aligned}
$$

where, for $q=\varrho_{1} / \varrho_{2}$,

$$
\begin{gathered}
\mathcal{N}=[B(E-F)+q A(D-F)]\left(B^{2}+q A^{2}\right)^{*}\left(1+|F|^{2}\right) \\
+\left[(E-F)^{2}+q(D-F)^{2}\right][B(E-F)+q A(D-F)]^{*}, \\
\mathcal{D}=\left|(E-F)^{2}+q(D-F)^{2}\right|^{2}-\left|B^{2}+q A^{2}\right|^{2}\left(1+|F|^{2}\right)^{2} .
\end{gathered}
$$

In order to have a large solar mixing angle, either $A q$ or $B$ must not be small compared to $E-F$ and $D-F$. But since $A, C \propto \frac{\mu_{2}}{\widetilde{\mu}_{2}}$, we are led to the case

$$
A=C \sim 0, \quad B=\frac{\rho_{2}}{\bar{\rho}_{2}} \frac{\widetilde{M_{1}}}{\widetilde{M}}=\mathcal{O}(1)
$$

Then we can neglect the terms proportional to $A$ and we have simply $\tan 2 \theta_{12}=2|B||E-F| \sqrt{1+|F|^{2}} \frac{|B|^{2}\left(1+|F|^{2}\right)+|E-F|^{2}+q(D-F)^{2} \frac{(E-F)^{*}}{E-F}}{\left|(E-F)^{2}+q(D-F)^{2}\right|^{2}-|B|^{4}\left(1+|F|^{2}\right)^{2}}$. 
This formula simplifies further if we neglect the $q(D-F)$ terms as well. ${ }^{4}$ Then using general trigonometric formulae leads to the expression in Eq. (4.11),

$$
\tan \theta_{12} \simeq \frac{|B|}{|E-F|} \sqrt{1+|F|^{2}}
$$

Taking the experimental value for the solar angle, $\tan ^{2} \theta_{12}=0.45 \pm 0.05$, gives us for $|F| \sim 1$ the range $|B| \sim(0.45-0.50)|E-F|$.

We can also compute the corrections of order $\varrho_{1,2}$ to the other two mixing angles, that we have discussed in the lowest order. In fact, since $\mu_{2} \ll \widetilde{\mu}_{2}$, the contribution (A.35) is small and the leading contribution to $\theta_{13}$ comes from the $B \varrho_{2}$ term,

$$
\left(V_{\nu}^{(1)}\right)_{13}=\sin \theta_{13} \simeq \frac{|B(E F+1)|}{\left(1+|F|^{2}\right)^{3 / 2}} \frac{\left|\varrho_{2}\right|}{\left|\varrho_{3}\right|} \sim|B| \frac{m_{2}}{m_{3}} \sim 0.2|B| .
$$

So even for vanishing leading order, we expect the first order term to bring $\theta_{13}$ near to the experimental bound. Note that it is the large solar angle that naturally gives $\theta_{13} \sim \varrho_{2} / \varrho_{3}$; in our model it seems pretty difficult to suppress this angle to much smaller values, apart if there is a tuned cancellation between zero and first order.

The corrections to the atmospheric angle are of the same order $\varrho_{2} / \varrho_{3}$ and do not have a large effect since we need in any case large parameters in the 23 sector. This small shift can in fact be easily compensated by a small change in the value of $F$, especially since we do not have any particular symmetry in the model imposing $F=1$.

Sum Rules for $B$ dominance and vanishing $\boldsymbol{m}_{\mathbf{1}}$. We have seen in the previous paragraph that in case of vanishing $C, A$ and $\varrho_{1}$, simple expressions can be obtained for all observables as functions of only few parameters $B, E, F$ and $\varrho_{3,2}$. Then it is possible to obtain relations between the different observables,

$$
\begin{aligned}
\tan \theta_{23} & =|F|, \\
\tan \theta_{12} & =\frac{|B|}{|E-F|} \sqrt{1+|F|^{2}}, \\
\sin \theta_{13} & =\frac{|B(E F+1)|}{\left(1+\left|\varrho_{2}\right|\right.} \frac{\left.\mid \varrho^{2}\right)^{3 / 2}}{\left|\varrho_{3}\right|}, \\
\frac{\delta m_{\mathrm{sol}}}{\delta m_{\mathrm{atm}}} & =\frac{\left|\varrho_{2}\right|}{\left|\varrho_{3}\right|} \frac{\sqrt{\left(1+|F|^{2}\right)^{2}\left(1+|E|^{2}+|B|^{2}\right)^{2}-|1-F E|^{4}}}{\left(1+|F|^{2}\right)^{2}} .
\end{aligned}
$$

\footnotetext{
${ }^{4}$ Note that taking $A=C=D-F=0$ gives a zero determinant for the neutrino mass matrix, so this case applies when the lightest eigenvalue is suppressed compared to the solar mass scale.
} 
Now we can write the following relation,

$$
\frac{\sin \theta_{13}}{\tan \theta_{12}} \frac{\delta m_{\mathrm{atm}}}{\delta m_{\mathrm{sol}}}=\frac{|E-F||E F+1|}{\sqrt{\left[\left(1+|F|^{2}\right)\left(1+|E|^{2}\right)+|E-F|^{2} \tan ^{2} \theta_{12}\right]^{2}-|1-E F|^{4}}} .
$$

To estimate its value, we can use the fact that $|F| \sim 1$ and vary only $|E|$ and the phases of $E, F$. We obtain then a maximal value of the r.h.s. for $E F=1$ so that

$$
\sin \theta_{13} \leq \frac{\delta m_{\mathrm{sol}}}{\delta m_{\mathrm{atm}}} \frac{\tan \theta_{12}}{1+\tan ^{2} \theta_{12}} \simeq 0.09
$$

Of course, the angle $\theta_{13}$ can always be reduced by an appropriate choice of the phases and in particular for $E=F$, so that there is no lower bound in this type of models.

The effective neutrino Majorana matrix, which is relevant for neutrinoless double beta decay, simplifies such that

$$
\begin{aligned}
\left|m_{e e}\right| & =|B|^{2}\left|\varrho_{2}\right| \\
& =\delta m_{\mathrm{sol}} \frac{\tan ^{2} \theta_{12}|E-F|^{2}}{\sqrt{\left[\left(1+|F|^{2}\right)\left(1+|E|^{2}\right)+\tan ^{2} \theta_{12}|E-F|^{2}\right]^{2}-|1-F E|^{4}}} .
\end{aligned}
$$

Again varying the phases and the modulus of $E$, we find the maximal value for $E F=-1$,

$$
\left|m_{e e}\right| \leq \delta m_{\text {sol }} \frac{\tan \theta_{12}}{\sqrt{2+\tan ^{2} \theta_{12}}} \sim 0.43 \delta m_{\text {sol }} .
$$

Moreover, we can give a simple relation between $m_{e e}$ and the reactor angle,

$$
\frac{\left|m_{e e}\right|}{\delta m_{\mathrm{atm}}}=\frac{|E-F|}{|E F+1|} \sin \theta_{13} \tan \theta_{12} .
$$

Note that the singular value for $E F+1=0$ corresponds to a vanishing reactor angle.

We can even derive a maximal value for the Dirac CP violation for this case. From Eqs. (4.21) and (4.27) we get

$$
\begin{aligned}
J_{\ell} & =-\frac{|B|^{2}\left(\kappa_{1}-\kappa_{2}\right) \operatorname{Im}(\Omega)}{\left(1+|F|^{2}\right)^{2}\left[\left(1+|F|^{2}\right)^{2}\left(1+|E|^{2}+|B|^{2}\right)^{2}-|1-E F|^{4}\right]} \\
& =-\frac{|E-F|^{4}}{1+|F|^{2}} \frac{\tan ^{2} \theta_{12}\left(1+\tan ^{2} \theta_{12}\right) \operatorname{Im}(\Omega)}{\left[\left(1+|F|^{2}\right)\left(1+|E|^{2}\right)+|E-F|^{2} \tan ^{2} \theta_{12}\right]^{2}-|1-E F|^{4}} \\
& =-\frac{\delta m_{\mathrm{sol}}}{\delta m_{\mathrm{atm}}} \frac{|E-F|^{4}}{1+|F|^{2}} \frac{\tan ^{2} \theta_{12}\left(1+\tan ^{2} \theta_{12}\right) \operatorname{Im}\left[\left(1+E F^{*}\right) F^{*}(E-F) e^{i \Delta_{23}}\right]}{\left[\left(\left(1+|F|^{2}\right)\left(1+|E|^{2}\right)+|E-F|^{2} \tan ^{2} \theta_{12}\right)^{2}-|1-E F|^{4}\right]^{3 / 2}},
\end{aligned}
$$


where $\Delta_{23}$ is the phase of $\varrho_{2} / \varrho_{3}$. Again, the prefactor is maximal for $E F=-1$ and $E=-F$, giving

$$
\left|J_{\ell}\right| \leq \frac{\delta m_{\text {sol }}}{\delta m_{\text {atm }}} \frac{1+\tan ^{2} \theta_{12}}{2 \tan \theta_{12}\left(2+\tan ^{2} \theta_{12}\right)^{3 / 2}}\left|\sin \Delta_{23}\right| \leq 0.06 .
$$

Here the imaginary part is only given by the phase $\Delta_{23}$, but in more general cases the phases of $E$ and $F$ will play a role as well. So even for the CP violation in the leptonic sector, the model displays a suppression given by the ratio of the mass eigenvalues. Contrary to the quark case, however, the $\mathrm{CP}$ violation is not proportional to the smallest mass eigenvalue, but it can be non-vanishing even for $m_{1}=0$.

\section{B. CP Violation and Weak Basis Invariants}

For completeness we discuss here the $\mathrm{CP}$ invariants in the case of an additional vectorial state. We prove that if the additional state is much heavier than the electroweak scale, the low energy CP violation can be expressed by the Jarlskog invariant defined from an effective $3 \times 3$ down quark mass matrix.

The transformation of a Dirac spinor $\psi(t, \vec{x})$ under parity and charge conjugation is given by

$$
\begin{aligned}
& \mathrm{P} \psi(t, \vec{x}) \mathrm{P}^{-1}=\eta_{P} \gamma^{0} \psi(t,-\vec{x}), \\
& \mathrm{C} \psi(t, \vec{x}) \mathrm{C}^{-1}=\eta_{C} C \bar{\psi}(t, \vec{x})^{\top},
\end{aligned}
$$

where $\eta_{P, C}$ are non-observable phases. The matrix $C$ obeys the relation $\gamma_{\mu} C=$ $-C \gamma_{\mu}^{T}$. Since the Lagrangian is a Lorentz scalar, it only depends on fermionic field bilinears. Thus, we deduce the CP transformation for such terms,

$$
\begin{aligned}
\mathrm{CP} \bar{\psi}_{i} \psi_{j}(\mathrm{CP})^{-1} & =\bar{\psi}_{j} \psi_{i}, \\
\mathrm{CP} \bar{\psi}_{i} \gamma^{5} \psi_{j}(\mathrm{CP})^{-1} & =-\bar{\psi}_{j} \gamma^{5} \psi_{i}, \\
\mathrm{CP} \bar{\psi}_{i} \gamma^{\mu} \psi_{j}(\mathrm{CP})^{-1} & =-\bar{\psi}_{j} \gamma_{\mu} \psi_{i}, \\
\mathrm{CP} \bar{\psi}_{i} \gamma^{\mu} \gamma^{5} \psi_{j}(\mathrm{CP})^{-1} & =-\bar{\psi}_{j} \gamma_{\mu} \gamma^{5} \psi_{i}
\end{aligned}
$$

Note that the operator $\partial_{\mu}$ transforms under CP as $\partial^{\mu} \rightarrow \partial_{\mu}$.

Quark Sector. In the Standard Model, it is easy to verify the existence of the CP symmetry in the Lagrangian, up to mass terms. In general, the quark mass terms are CP invariant if and only if it is possible to find a weak basis transformation which realises

$$
H^{u *}=W_{L} H^{u} W_{L}^{\dagger}, \quad H^{d *}=W_{L} H^{d} W_{L}^{\dagger},
$$

where $H^{u, d}=M^{u, d}\left(M^{u, d}\right)^{\dagger}$. It follows that

$$
W_{L}\left[H_{u}, H_{d}\right] W_{L}^{\dagger}=-\left[H_{u}, H_{d}\right]^{\top}
$$


such that, for $r$ odd,

$$
\operatorname{tr}\left[H_{u}, H_{d}\right]^{r}=0
$$

is a necessary and sufficient condition for CP invariance [42].

The case of $r=1$ is trivial: the trace of a commutator $\left[H_{u}, H_{d}\right]$ is zero. For $r=3$ and three generations, we have

$$
\begin{aligned}
I_{\mathrm{SM}} \equiv \operatorname{tr}\left[H_{u}, H_{d}\right]^{3}=6 i & \left(m_{t}^{2}-m_{c}^{2}\right)\left(m_{t}^{2}-m_{u}^{2}\right)\left(m_{c}^{2}-m_{u}^{2}\right) \\
& \left(m_{b}^{2}-m_{s}^{2}\right)\left(m_{b}^{2}-m_{d}^{2}\right)\left(m_{s}^{2}-m_{d}^{2}\right) J_{q}
\end{aligned}
$$

where the quantity $J_{q}$ does not depend of the mass spectrum, and can be related, up to a sign, with the CKM matrix, $V$, as $\left|J_{q}\right|=\left|\operatorname{Im}\left(V_{12} V_{13}^{*} V_{22}^{*} V_{23}\right)\right|$. We conclude that in order to have CP violation, we need to have $J_{q} \neq 0$. This quantity is the lowest weak basis invariant which measure $\mathrm{CP}$ violating effects and it has mass-dimension twelve. Apart from $\mathrm{CP}$ violation in the strong interactions, there is no other mechanism in the SM which can generate CP violating effects if $J_{q}=0$. Note that in the chiral limit, $m_{u}=m_{d}=m_{s}=0$, we do not generate $\mathrm{CP}$ violation even if $J_{q} \neq 0$.

In the literature, the lowest weak basis invariant is called Jarlskog determinant [28],

$$
\begin{aligned}
\operatorname{det}\left[H_{u}, H_{d}\right]=2 i & \left(m_{t}^{2}-m_{c}^{2}\right)\left(m_{t}^{2}-m_{u}^{2}\right)\left(m_{c}^{2}-m_{u}^{2}\right) \\
& \left(m_{b}^{2}-m_{s}^{2}\right)\left(m_{b}^{2}-m_{d}^{2}\right)\left(m_{s}^{2}-m_{d}^{2}\right) J_{q} .
\end{aligned}
$$

which is equivalent to the Eq. (B.6).$^{5}$ The Jarlskog determinant is only applicable to the case of three generations, in contrast to the more general invariant in Eq. (B.5).

Now let us add a down quark isosinglet. The gauge couplings to quarks and their mass terms are $(i, j=1,2,3$ and $\alpha=1,2,3,4)$ :

$$
\begin{aligned}
\mathscr{L}_{W}^{q}= & -\frac{g}{\sqrt{2}}\left(\bar{u}_{L i} \gamma^{\mu} d_{L i} W_{\mu}^{+}+\text {h.c. }\right)-e J_{\mathrm{EM}}^{\mu} A_{\mu} \\
& -\frac{g}{2 \cos \theta_{\mathrm{W}}}\left(\bar{u}_{L i} \gamma^{\mu} u_{L i}-\bar{d}_{L i} \gamma^{\mu} d_{L i}-2 \sin ^{2} \theta_{\mathrm{W}} J_{\mathrm{EM}}^{\mu}\right) Z_{\mu} \\
\mathscr{L}_{M}^{q}= & -\left(\bar{u}_{L i} M_{u}^{i j} u_{R j}+\bar{d}_{L i} M_{d}^{i \alpha} d_{R \alpha}+\bar{d}_{L 4} m_{d}^{\alpha} d_{R \alpha}\right)+\text { h.c. }
\end{aligned}
$$

where the matrices $M_{u}, M_{d}$ and $m_{d}$ are of dimension $3 \times 3,3 \times 4$ and $1 \times 4$, respectively. The electromagnetic current is given by $J_{\mathrm{E} M}^{\mu}=\frac{2}{3} \bar{u} \gamma^{\mu} u-\frac{1}{3} \bar{d} \gamma^{\mu} d$.

The most general weak basis transformation consistent with the Lagrangian of Eq. (B.8) is:

$$
\left(\begin{array}{c}
u_{L i} \\
d_{L i}
\end{array}\right) \longrightarrow U_{L}^{i j}\left(\begin{array}{c}
u_{L j} \\
d_{L j}
\end{array}\right), \quad u_{R i} \longrightarrow\left(U_{R}^{u}\right)^{i j} u_{R j}, \quad d_{R \alpha} \longrightarrow\left(U_{R}^{d}\right)^{\alpha \beta} d_{R \alpha}
$$

\footnotetext{
${ }^{5}$ For any $3 \times 3$ traceless Hermitian matrix $M$ one has: $\operatorname{tr} M^{3}=3|M|$.
} 
where $U_{L}$ and $U_{R}^{u}$ are $3 \times 3$ unitary matrices, while $U_{R}^{d}$ is $4 \times 4$. Once we diagonalise the mass terms, the Lagrangian reads

$$
\begin{aligned}
\mathscr{L}_{W}= & -\frac{g}{\sqrt{2}}\left[\bar{u}_{L i} \gamma^{\mu}\left(V_{\mathrm{CKM}}\right)_{i \alpha} d_{L \alpha} W_{\mu}^{+}+\text {h.c. }\right]-e J_{\mathrm{EM}}^{\mu} A_{\mu} \\
& -\frac{g}{2 \cos \theta_{\mathrm{W}}}\left[\bar{u}_{L i} \gamma^{\mu} u_{L i}-\bar{d}_{L \alpha} \gamma^{\mu}\left(V_{\mathrm{CKM}}^{\dagger} V_{\mathrm{CKM}}\right)_{\alpha \beta} d_{L \beta}-2 \sin ^{2} \theta_{\mathrm{W}} J_{\mathrm{EM}}^{\mu}\right] Z_{\mu}, \\
\mathscr{L}_{M}= & -\left(\bar{u}_{L i} D_{u i} u_{R i}+\bar{d}_{L \alpha} D_{d \alpha} d_{R \alpha}\right)+\text { h.c. },
\end{aligned}
$$

where $V_{\mathrm{CKM}}=U_{L}^{u \dagger} U_{L}^{d}$ is a $3 \times 4$ matrix. The number of independent phases which are related to $\mathrm{CP}$ violation is, for $N=3$,

$$
n_{\mathrm{CP}}=N(N+1)-\frac{1}{2} N(N-1)-2 N=\frac{1}{2} N(N-1)=3 .
$$

With the matrices as defined in Eq. (B.8b) and $H_{u}=M_{u} M_{u}^{\dagger}, H_{d}=M_{d} M_{d}^{\dagger}$, and $h_{d}=M_{d} m_{d}^{\dagger}$, we can write down a set of weak basis invariants,

$$
\begin{array}{lll}
I_{1}=\operatorname{Im} \operatorname{tr} H_{u} H_{d} h_{d} h_{d}^{\dagger}, & I_{2}=\operatorname{Im} \operatorname{tr} H_{u}^{2} H_{d} h_{d} h_{d}^{\dagger}, \\
I_{3}=\operatorname{Im} \operatorname{tr} H_{u}^{2}\left[H_{u}, H_{d}\right] h_{d} h_{d}^{\dagger}, & I_{4}=\operatorname{Im} \operatorname{tr} H_{u} H_{d}^{2} h_{d} h_{d}^{\dagger}, \\
I_{5}=\operatorname{Im} \operatorname{tr} H_{u}^{2} H_{d}^{2} h_{d} h_{d}^{\dagger}, & I_{6}=\operatorname{Im} \operatorname{tr} H_{u}^{2}\left[H_{u}, H_{d}^{2}\right] h_{d} h_{d}^{\dagger}, \\
I_{7}=\operatorname{Im} \operatorname{tr} H_{u}^{2} H_{d} H_{u} H_{d}^{2}, &
\end{array}
$$

representing a set of necessary and sufficient conditions for having $\mathrm{CP}$ invariance in the quark sector [43].

In our model, $H_{d}$ and $h_{d}$ read

$$
H_{d}=\left(\begin{array}{ccc}
\left|\mu_{1}\right|^{2}+\widetilde{\mu}_{1}^{2} & \widetilde{\mu}_{1} \widetilde{\mu}_{2} & \widetilde{\mu}_{1} \widetilde{\mu}_{3} \\
\widetilde{\mu}_{1} \widetilde{\mu}_{2} & \left|\mu_{2}\right|^{2}+\widetilde{\mu}_{2}^{2} & \widetilde{\mu}_{2} \widetilde{\mu}_{3} \\
\widetilde{\mu}_{1} \widetilde{\mu}_{3} & \widetilde{\mu}_{2} \widetilde{\mu}_{3} & \left|\mu_{3}\right|^{2}+\widetilde{\mu}_{3}^{2}
\end{array}\right), \quad h_{d}=\left(\begin{array}{c}
\mu_{1} \widetilde{M}_{1}+\widetilde{\mu}_{1} \widetilde{M}_{4} \\
\mu_{2} \widetilde{M}_{2}+\widetilde{\mu}_{2} \widetilde{M}_{4} \\
\mu_{3} \widetilde{M}_{3}+\widetilde{\mu}_{3} \widetilde{M}_{4}
\end{array}\right)
$$

Since $H_{u}$ and $H_{d}$ are real, $I_{7}$ vanishes. The remaining invariants are in general different from zero; the dominant terms are

$$
\begin{array}{ll}
I_{1}=-m_{t}^{2}\left(\widetilde{\mu}_{1}^{2}+\widetilde{\mu}_{2}^{2}\right) \widetilde{\mu}_{3} \widetilde{M}_{4} \operatorname{Im} \mu_{3}, & I_{2}=m_{t}^{2} I_{1}, \\
I_{3}=-m_{t}^{6}\left(\widetilde{\mu}_{1}^{2}+\widetilde{\mu}_{2}^{2}\right) \widetilde{\mu}_{3} \widetilde{M}_{3} \widetilde{M}_{4} \operatorname{Im} \mu_{3}, & I_{5}=m_{t}^{2} I_{4}, \\
I_{4}=-m_{t}^{2}\left(\widetilde{\mu}_{1}^{2}+\widetilde{\mu}_{2}^{2}\right)\left(\widetilde{\mu}_{1}^{2}+\widetilde{\mu}_{2}^{2}+\widetilde{\mu}_{3}^{2}+\mu_{3}^{2}\right) \widetilde{\mu}_{3} \widetilde{M}_{3} \widetilde{M}_{4} \operatorname{Im} \mu_{3}, & \\
I_{6}=-m_{t}^{6}\left(\widetilde{\mu}_{1}^{2}+\widetilde{\mu}_{2}^{2}\right)\left(\widetilde{\mu}_{1}^{2}+\widetilde{\mu}_{2}^{2}+\widetilde{\mu}_{3}^{2}+\mu_{3}^{2}\right) \widetilde{\mu}_{3} \widetilde{M}_{3} \widetilde{M}_{4} \operatorname{Im} \mu_{3} . &
\end{array}
$$

Hence, CP is generally violated even by the presence of a single complex parameter $\mu_{3}$. Note that this case is not equivalent to the chiral limit because both the charm and strange masses are different from zero, $m_{c} \propto \mu_{2}$ and $m_{s} \sim \widetilde{\mu}_{2}$ (albeit $\mu_{2} \ll$ 
$\widetilde{\mu}_{2}$ ). As we might expect, the invariants vanish if all quarks of the first and second generation are massless.

Now we single out the heavy eigenstate with the rotations $V_{4}, U_{4}$. While the action of $V_{4}$ leaves the invariants unaffected, $U_{4}$ strongly modifies them and reshuffles terms from one to the other. In fact after this transformation, $h_{d}$ vanishes to lowest order and survives only at order $\mathcal{O}\left(v_{E W}^{2} / \widetilde{M}^{2}\right)$; then in the new basis all the invariants involving $h_{d}$, i.e., $I_{1}-I_{6}$ are suppressed by $v_{\mathrm{EW}}^{2} / \widetilde{M}^{2}$ and vanish for $\widetilde{M} \rightarrow \infty$. On the other hand $I_{7}$ is now non-vanishing and given by

$$
I_{7}^{\prime}=\operatorname{Im} \operatorname{tr} H_{u}^{2} H_{d}^{\mathrm{eff}} H_{u}\left(H_{d}^{\mathrm{eff}}\right)^{2},
$$

where $H_{d}^{\text {eff }}=\widehat{m} \widehat{m}^{\dagger}$ (see Eq. (2.7)). Note that $U_{4}$ also changes the weak interactions,

$$
\delta \mathscr{L}_{W}=-\frac{g}{\sqrt{2}} \bar{u}_{i} \gamma^{\mu}\left(U_{4}-\mathbb{1}\right)_{i 4} d_{4} W_{\mu}^{+}+\bar{d}_{i} \gamma^{\mu}\left(U_{4}^{\dagger} U_{4}-\mathbb{1}\right)_{i 4} d_{4} Z_{\mu}+\text { h.c. },
$$

so we expect both $\mathrm{CP}$ violation and CKM unitarity violation from these terms as well. However, the mass of the heavy state is $\mathcal{O}\left(M_{\mathrm{GUT}}\right)$ so that the contributions to low-energy processes are suppressed by a factor $M_{\mathrm{EW}} / M_{\mathrm{GUT}}$ and are negligible.

Hence, at the electroweak scale, we are left to consider the single invariant

$$
I_{7}^{\prime}=\operatorname{Im} \operatorname{tr} H_{u}^{2} H_{d}^{\mathrm{eff}} H_{u} H_{d}^{\mathrm{eff} 2}
$$

which corresponds to the usual Jarlskog invariant $J_{q}$ for three generations, but computed for the effective quark mass $\widehat{m}$.

Lepton Sector. As discussed above, we can ignore the heavy states for low-energy $\mathrm{CP}$ violation and use the effective $3 \times 3$ Yukawa matrices instead.

In the SM, extended by right-handed neutrinos, we have three mass terms for the leptons,

$$
\mathscr{L}_{M}^{\ell}=-\left(\bar{e}_{L i} m_{e}^{i j} e_{R j}+\bar{\nu}_{L i} m_{D}^{i j} \nu_{R j}+\frac{1}{2} \nu_{R i}^{\top} C m_{N}^{i j} \nu_{R j}\right)+\text { h.c. }
$$

In analogy to the quark sector, invariance of the mass terms under CP transformation requires

$$
U^{\dagger} m_{e} V=m_{e}^{*}, \quad U^{\dagger} m_{D} W=m_{D}^{*}, \quad W^{\top} m_{N} W=-M_{R}^{*},
$$

where $U, V$, and $W$ are unitary matrices acting in flavour space. Defining $h=m_{D}^{\dagger} m_{D}$ and $H=m_{N}^{\dagger} m_{N}$, we obtain

$$
W^{\dagger} h W=h^{*}, \quad W^{\dagger} H W=H^{*} .
$$

Now we can write down the weak basis invariants

$$
\begin{aligned}
& I_{1}^{\ell}=\operatorname{Im} \operatorname{tr} h H m_{N}^{*} h^{*} m_{N}, \quad I_{2}^{\ell}=\operatorname{Im} \operatorname{tr} h H^{2} m_{N}^{*} h^{*} m_{N}, \\
& I_{3}^{\ell}=\operatorname{Im} \operatorname{tr} h H^{2} m_{N}^{*} h^{*} m_{N} H \text {; }
\end{aligned}
$$


for the three further invariants, substitute $\bar{h}=m_{D}^{\dagger} m_{e} m_{e}^{\dagger} m_{D}$ for $h$ [26]. In the basis where the right-handed neutrino mass is diagonal, one obtains

$$
\begin{aligned}
& I_{1}^{\ell}=M_{1} M_{2}\left(M_{2}^{2}-M_{1}^{2}\right) \operatorname{Im} h_{12}^{2} \\
& \quad+M_{1} M_{3}\left(M_{3}^{2}-M_{1}^{2}\right) \operatorname{Im} h_{13}^{2}+M_{2} M_{3}\left(M_{3}^{2}-M_{2}^{2}\right) \operatorname{Im} h_{23}^{2}, \\
& \begin{aligned}
I_{2}^{\ell}= & M_{1} M_{2}\left(M_{2}^{4}-M_{1}^{4}\right) \operatorname{Im} h_{12}^{2} \\
\quad & \quad M_{1} M_{3}\left(M_{3}^{4}-M_{1}^{4}\right) \operatorname{Im} h_{13}^{2}+M_{2} M_{3}\left(M_{3}^{4}-M_{2}^{4}\right) \operatorname{Im} h_{23}^{2}, \\
I_{3}^{\ell}= & M_{1}^{3} M_{2}^{3}\left(M_{2}^{2}-M_{1}^{2}\right) \operatorname{Im} h_{12}^{2} \\
& \quad+M_{1}^{3} M_{3}^{3}\left(M_{3}^{2}-M_{1}^{2}\right) \operatorname{Im} h_{13}^{2}+M_{2}^{3} M_{3}^{3}\left(M_{3}^{2}-M_{2}^{2}\right) \operatorname{Im} h_{23}^{2} .
\end{aligned}
\end{aligned}
$$

If none of the $M_{i}$ vanish and there is no degeneracy, the vanishing of $I_{1}, I_{2}$, and $I_{3}$ implies the vanishing of $\operatorname{Im} h_{12}^{2}, \operatorname{Im} h_{13}^{2}$, and $\operatorname{Im} h_{23}^{2}$ for CP invariance.

Note that in our model, $m_{D}$ stands for the effective $3 \times 3$ part of the Dirac neutrino mass matrix, $\bar{m}_{D}$, as given in Eq. (A.23). Then we obtain from Eq. (4.3),

$$
\begin{aligned}
& h_{12}=A^{*} D \bar{\rho}_{1}^{2}+B^{*} E \bar{\rho}_{2}^{2}+C^{*} F \bar{\rho}_{3}^{2}, \\
& h_{13}=A \bar{\rho}_{1}^{2}+B \bar{\rho}_{2}^{2}+C \bar{\rho}_{3}^{2}, \\
& h_{23}=D^{*} \bar{\rho}_{1}^{2}+E^{*} \bar{\rho}_{2}^{2}+F^{*} \bar{\rho}_{3}^{2} .
\end{aligned}
$$

The coefficients $A, \ldots, F$ are displayed in Eqs. (A.24). They are generically complex, so we do not expect CP to be conserved.

As in the quark sector, these invariants are rather general and give the necessary conditions for the presence of $\mathrm{CP}$ violation. On the other hand, only few of the phases remain important also in the low-energy limit. In our case, to study the low-energy Dirac invariant, we can use the analogue of the Jarlskog invariant,

$$
J_{\ell}=-\frac{1}{\mathscr{M}_{\nu}^{2} \mathscr{M}_{e}^{2}} \operatorname{tr}\left[h_{\mathrm{eff}}^{\nu}, h^{e}\right]^{3},
$$

as discussed in Section 4.3. Here, $h_{\text {eff }}^{\nu}=\left(m_{\text {eff }}^{\nu}\right)^{\dagger} m_{\text {eff }}^{\nu}$ and $\Delta \mathscr{M}_{\nu}^{2}$ and $\Delta \mathscr{M}_{e}^{2}$ are the products of the mass squared differences of the light neutrinos and charged leptons, respectively. 


\section{References}

[1] H. Fritzsch and Z. Z. Xing, Mass and flavor mixing schemes of quarks and leptons, Prog. Part. Nucl. Phys. 45 (2000) 1 hep-ph/9912358.

[2] G. G. Ross, Models of fermion masses, in Theoretical Advanced Study Institute in Elementary Particle Physics (TASI 2000): Flavor Physics for the Millennium, Boulder, Colorado, 4-30 Jun 2000.

[3] R. N. Mohapatra and A. Y. Smirnov, Neutrino mass and new physics, Ann. Rev. Nucl. Part. Sci. 56 (2006) 569 hep-ph/0603118].

[4] G. Altarelli, Models of neutrino masses and mixings, hep-ph/0611117.

[5] H. Georgi, Unified Gauge Theories, in Coral Gables 1975, Proceedings, Theories and Experiments In High Energy Physics, New York 1975, 329-339.

[6] H. Fritzsch and P. Minkowski, Unified Interactions Of Leptons And Hadrons, Ann. Phys. (NY) 93 (1975) 193.

[7] Y. Kawamura, Triplet doublet splitting, proton stability and extra dimension, Prog. Theor. Phys. 105 (2001) 999 hep-ph/0012125.

[8] G. Altarelli and F. Feruglio, SU(5) grand unification in extra dimensions and proton decay, Phys. Lett. B 511 (2001) 257 hep-ph/0102301.

[9] L. J. Hall and Y. Nomura, Gauge unification in higher dimensions, Phys. Rev. D 64 (2001) 055003 hep-ph/0103125.

[10] A. Hebecker and J. March-Russell, A Minimal $S^{1} /\left(Z_{2} \times Z_{2}^{\prime}\right)$ Orbifold GUT, Nucl. Phys. B 613 (2001) 3 hep-ph/0106166.

[11] T. Asaka, W. Buchmüller, and L. Covi, Gauge unification in six dimensions, Phys. Lett. B 523 (2001) 199 hep-ph/0108021.

[12] L. J. Hall, Y. Nomura, T. Okui, and D. R. Smith, SO(10) unified theories in six dimensions, Phys. Rev. D 65 (2002) 035008 hep-ph/0108071.

[13] T. Asaka, W. Buchmüller, and L. Covi, Quarks and leptons between branes and bulk, Phys. Lett. B 563 (2003) 209 hep-ph/0304142.

[14] W. Buchmüller, L. Covi, D. Emmanuel-Costa and S. Wiesenfeldt, Flavour structure and proton decay in 6D orbifold GUTs, J. High Energy Phys. 09 (2004) 004 hep-ph/0407070.

[15] W. Buchmüller, J. Kersten and K. Schmidt-Hoberg, Squarks and sleptons between branes and bulk, J. High Energy Phys. 0602 (2006) 069 hep-ph/0512152.

[16] H. M. Lee, Gauge coupling unification in six dimensions, Phys. Rev. D 75 (2007) 065009 [Erratum-ibid. D 76 (2007) 029902] hep-ph/0611196.

[17] H. D. Kim, S. Raby and L. Schradin, Quark and lepton masses in 5D SO(10), J. High Energy Phys. 0505 (2005) 036 hep-ph/0411328.

[18] G. Bhattacharyya, G. C. Branco and J. I. Silva-Marcos, CP Violation and Flavour Mixings in Orbifold GUTS, arXiv:0709.1848 [hep-ph].

[19] S. M. Barr, Light Fermion Mass Hierarchy And Grand Unification, Phys. Rev. D 21 (1980) 1424. 
[20] Y. Nomura and T. Yanagida, Bi-maximal neutrino mixing in SO(10) GUT, Phys. Rev. D 59 (1999) 017303 hep-ph/9807325.

[21] T. Asaka, Lopsided mass matrices and leptogenesis in SO(10) GUT, Phys. Lett. B 562 (2003) 291 hep-ph/0304124.

[22] W. Buchmüller, K. Hamaguchi, O. Lebedev, and M. Ratz, Supersymmetric standard model from the heterotic string, Phys. Rev. Lett. 96 (2006) 121602

hep-ph/0511035; Supersymmetric Standard Model from the Heterotic String (II), Nucl. Phys. B 785 (2007) 149 hep-th/0606187.

[23] O. Lebedev, H. P. Nilles, S. Raby, S. Ramos-Sanchez, M. Ratz, P. K. S. Vaudrevange and A. Wingerter, A mini-landscape of exact MSSM spectra in heterotic orbifolds, Phys. Lett. B 645 (2007) 88 hep-th/0611095|; The Heterotic Road to the MSSM with $R$ parity, arXiv:0708.2691 [hep-th].

[24] W. Buchmüller, C. Lüdeling and J. Schmidt, Local SU(5) Unification from the Heterotic String, J. High Energy Phys. 0709 (2007) 113 arXiv:0707.1651 [hep-ph]].

[25] T. Asaka, W. Buchmüller, L. Covi, Bulk and brane anomalies in six dimensions, Nucl. Phys. B 648 (2003) 231 hep-ph/0209144.

[26] G. C. Branco, T. Morozumi, B. M. Nobre and M. N. Rebelo, A bridge between CP violation at low energies and leptogenesis, Nucl. Phys. B 617 (2001) 475 hep-ph/0107164.

[27] G. C. Branco and M. N. Rebelo, Leptonic CP violation and neutrino mass models, New J. Phys. 7 (2005) 86 hep-ph/0411196.

[28] C. Jarlskog, Commutator of the Quark Mass Matrices in the Standard Electroweak Model and a Measure of Maximal CP Violation, Phys. Rev. Lett. 55 (1985) 1039.

[29] W. M. Yao et al. [Particle Data Group], Review of particle physics, J. Phys. G 33 (2006)1.

[30] F. Vissani, Large mixing, family structure, and dominant block in the neutrino mass matrix, J. High Energy Phys. 11 (1998) 025 hep-ph/9810435.

[31] M. C. Gonzalez-Garcia and M. Maltoni, Phenomenology with Massive Neutrinos, arXiv:0704.1800 [hep-ph].

[32] S. T. Petcov, Neutrino Masses, Mixing, Majorana CP-Violating Phases And $(\beta \beta)(0 \nu)$ Decay, New J. Phys. 6 (2004) 109.

[33] M. Fukugita and T. Yanagida, Baryogenesis Without Grand Unification, Phys. Lett. B 174 (1986) 45.

[34] L. Covi, E. Roulet and F. Vissani, CP violating decays in leptogenesis scenarios, Phys. Lett. B 384 (1996) 169 hep-ph/9605319.

[35] W. Buchmüller and M. Plumacher, Baryon asymmetry and neutrino mixing, Phys. Lett. B 389 (1996) 73 hep-ph/9608308.

[36] W. Buchmüller and T. Yanagida, Quark lepton mass hierarchies and the baryon asymmetry, Phys. Lett. B 445 (1999) 399 hep-ph/9810308.

[37] W. Buchmüller, P. Di Bari and M. Plümacher, Leptogenesis for pedestrians, Ann. Phys. (NY) 315 (2005) 305 hep-ph/0401240. 
[38] A. Abada, S. Davidson, F. X. Josse-Michaux, M. Losada and A. Riotto, Flavour issues in leptogenesis, JCAP $\mathbf{0 6 0 4}$ (2006) 004 hep-ph/0601083.

[39] E. Nardi, Y. Nir, E. Roulet and J. Racker, The importance of flavor in leptogenesis, J. High Energy Phys. 0601 (2006) 164 hep-ph/0601084.

[40] S. Blanchet, P. Di Bari and G. G. Raffelt, Quantum Zeno effect and the impact of flavor in leptogenesis, JCAP 0703 (2007) 012 hep-ph/0611337.

[41] S. Antusch, S. F. King and A. Riotto, Flavour-dependent leptogenesis with sequential dominance, JCAP 0611 (2006) 011 hep-ph/0609038.

[42] J. Bernabeu, G. C. Branco and M. Gronau, CP Restrictions On Quark Mass Matrices, Phys. Lett. B 169 (1986) 243.

[43] F. del Aguila, J. A. Aguilar-Saavedra and G. C. Branco, CP violation from new quarks in the chiral limit, Nucl. Phys. B 510 (1998) 39 hep-ph/9703410. 